\title{
A numerical model suggests the interplay between nuclear plasticity and stiffness during a perfusion assay
}

\author{
Solenne Deveraux ${ }^{\mathrm{a}}$, Rachele Allena ${ }^{\mathrm{b}}$, Denis Aubry ${ }^{\mathrm{a}}$ \\ ${ }^{a}$ Laboratoire MSSMat UMR CNRS 8579, CentraleSupelec, Université Paris-Saclay, Grande \\ Voie des Vignes, 92290 Châtenay-Malabry - France \\ ${ }^{b}$ Arts et Metiers Paris Tech, LBM/Institut de Biomécanique Humaine Georges Charpak, 151 \\ bd de l'Hôpital 75013 Paris - France
}

\begin{abstract}
\end{abstract}
Cell deformability is a necessary condition for a cell to be able to migrate, an ability that is vital both for healthy and diseased organisms. The nucleus being the largest and stiffest organelle, it often is a barrier to cell migration. It is thus essential to characterize its mechanical behaviour. First, we numerically investigate the visco-elasto-plastic properties of the isolated nucleus during a compression test. This simulation highlights the impact of the mechanical behaviour of the nuclear lamina and the nucleoplasm on the overall plasticity. Second, a whole cell model is developed to simulate a perfusion experiment to study the possible interactions between the cytoplasm and the nucleus. We analyze and discuss the role of the lamina for a wild-type cell model, and a lamin-deficient one, in which the Young's modulus of the lamina is set to $1 \%$ of its nominal value. Thís simulation suggests an interplay between the cytoplasm and the nucleoplasm, especially in the lamin-deficient cell, showing the need of a stiffer nucleoplasm to maintain nuclear plasticity.

Keywords: Nucleus, Plasticity, Finite Element, Viscoelasticity, Viscoplasticity

*Corresponding author

Email address: solenne.deveraux@centralesupelec.fr (Solenne Deveraux) 


\section{Introduction}

Cell motility is a fundamental cellular mechanism involved in several biological phenomena such as bone remodelling, immune response and tumor metastasis. Tumor metastasis is estimated to be responsible for $90 \%$ of cancer death [1]. In such cases, where cell migration through sub-nuclear pores is often necessary, the nucleus plays a critical role due to its size and mechanical properties [2]. Hence, a better understanding of the processes behind cell migration and of nuclear mechanics is primordial in order to develop new therapeutic strategies in the fight against cancer. Most publications investigate the molecular mechanisms of cancer metastasis [3], of mechanical properties of the nucleus [4] and of the mechanical coupling between the nucleus and the cell's cytoskeleton $[5,6]$ : the aim here is to tackle these issues through a mechanical perspective. During migration or transmigration events, the cell is capable of going through narrow constrictions down to $10 \%$ of the size of the nucleus [7]. The large size and high stiffness of the nucleus make it a major obstacle to this process, although some cells are able to overcome such difficulties [6]. Its mechanical properties mainly arise from two components: the lamina - a dense meshwork composed of A-type and B-type lamins, as well as lamin-associated proteins [8] - and the nucleoplasm. The nucleoplasm is mostly made up of chromatin surrounded by fluid and can thus be seen as a viscoelastic material. The lamina however, as a dense meshwork where fluid cannot circulate, can be seen as a solid elastic material [9]. With sheer observation of its internal organization, we can already qualitatively propose a visco-elastic model of the nucleus. Since nuclear mechanics is at stake here, numerical simulation appears as a very interesting tool to investigate the mechanical interplay between cellular components, such as the lamina and the cytoplasm, and to get new insights on some biological assumptions. As for quantitative values, many techniques are accessible to study specific features of the nucleus, as will be discussed in the following paragraphs. 


\subsection{From experimentation to mechanical modelling}

A wide range range of experimental techniques are available to investigate mechanical properties of various components of the cell and of the nucleus at various scales [10]. They include perfusion [11], micropipette aspiration - possibly coupled with relaxation experiments - [12], Atomic Force Microscopy (AFM), active or passive micro-rheology through magnetic or optical tweezers with local information [13], microplate compression [14], substrate strain, micro-needle manipulation [15], shear flow and cytoindentation [16]. The first experimental setups focused on getting the mechanical behaviour of the overall nucleus. Depending on the technique, different behaviours at various scales can be studied. The overall nucleus. The nucleus has widely been found to behave as a viscoelastic material, all throughout different techniques that enlightened various specific aspects of its behaviour [17, 18]. The perfusion and aspiration assays give a good assessment of purely passive mechanical properties of both the cell and the nucleus since they are fast enough-cells pass through in less than a second $[11,19,20]$ - to assume no cyto- or nucleoskeleton reorganization occurs. Such assays showed that nucleus was 3-4 times stiffer and twice more viscous than the cytoplasm $[12,14]$. The viscoelasticity of the nucleus is mostly accepted, but a hyperelastic behaviour of the nucleus is sometimes assumed in order to fit experimental data with simulation ones and to obtain quantitative data on the mechanical parameters of the nucleus [14]. Using these global measurement techniques, the Young modulus of the cell nucleus is estimated around 1-8 $\mathrm{kPa}[14,21,12,22]$, but is sometimes found to be much higher depending on the experimental method [23]. This illustrates one of the limits of the global techniques that raise various uncertainties due to the interaction between the nucleus and the rest of the cell. Some recent advances in AFM techniques are used to get rid of this bias by probing the nucleus more locally [22].

The nuclear envelope (NE) and the lamina. Information on the whole nucleus is essential and easier to get, but the tight interaction between mechanical forces 
and gene regulation induces to look more closely and precisely at local properties of the nucleus at the scale of specific proteins such as chromatin and lamins. Studies of the nuclear envelope alone are scarce, but combined techniques of micropipette aspiration and confocal microscopy were used to characterize the nuclear envelope as purely elastic [24]. Underlying the nuclear envelope is the lamina, a stiff material ensuring the nuclear stability, sometimes described as viscoelastic, although more thorough and velocity-dependent testing would be necessary to rigorously prove the viscoelastic behaviour $[25,26]$. Together, the lamina and the nuclear envelope form a very thin layer of 10-200 nm surrounding the nucleus [27]. Given the very high stiffness of the lamina, the impact of the NE, as well as lamina's viscosity can be neglected. Such stiffness protects the cell and its genetic information, but can also be a rate-limiting factor during confined migration by preventing sufficient nucleus deformation. In fact, cells have to find a good compromise between viability and motility.

The nucleoplasm. The nucleoplasm behaves as a sponge-like material that initially does not present much resistance to deformation but this resistance increases as the chromatin gets compacted [21]. Besides, chromatin exhibits a plastic behaviour, i.e. irreversible deformation, at long time-scales and after shear stresses, which decreases with up-regulation of Lamin-A [28, 29]. This suggests that the nucleoplasm sets the rheological character of the nucleus while the lamina dictates the extent of the deformation. Such plasticity is an advantage during confined migration, since the nucleus stays elongated after going through a narrowing space, making it easier for the cell to migrate once the first constriction is overcome. Most often, the lamina is considered as the main load-bearing element in the nucleus, but recent findings suggest that chromatin itself is the main structural component of the nucleus [30].

Existing computational models of the nucleus and its mechanical behaviour. The interest for cell computational models has been rising in the last two decades, as it becomes more and more obvious that mechanics plays a major role during cell migration and even in gene transcription. One strategy for representing the 
cell and the nucleus is discrete modelling, just considering the cell membrane or the nuclear envelope [31], another is an energetic approach [32], and finally, the cell or nucleus can be modeled through continuum mechanics [33]. Most continuum models describe the whole cell, with or without its nucleus as a separate compartment, but fewer model focus on the isolated nucleus, as reviewed in [34] and [16]. Our approach here is to propose a model which can be employed for both the isolated nucleus and the whole cell. The nucleus, if proven to be viscoelastic, is sometimes modeled as a hyperelastic material in order to simplify the simulation and to investigate specific mechanical issues [14, 35, 32].) A viscoelastic model was later developed to simulate a micro-pipette aspiration assay [36], with the lamina and nuclear envelope taken into account. A more advanced model was proposed with the nucleus described as a poroelastic material with a plastic behaviour [37]. Interestingly, this model faithfully reproduced the irreversible deformation found in Lamin $\mathrm{A} / \mathrm{C}$ deficient cells after transmigration. Although we acknowledge the validity and the interest of all these models, we observe that each one of them is designed to fit a specific experiment and can wonder whether one single model could describe several different assays. This is specifically what we aim to tackle in this article: a unified model of the whole cell that can be confronted with various experimental techniques.

\subsection{The proposed model}

Given the major role of nuclear mechanics during confined cell migration, this paper will present a two dimensions (2D) FE implementation of a cell nucleus model, representing the nuclear lamina as elastic and the nucleoplasm as visco-elasto-plastic. Even though a 3D model would be more accurate, we chose a $2 \mathrm{D}$ representation to facilitate the computation, since it was shown that for a cell entering a micro-channel, the model is insensitive to depth [38]. While the whole nucleus is generally described as merely viscoelastic, we decided to design a new model to be able to account for a more complex behaviour of the nucleus including plasticity. Besides, we aim at developing a model that is able to be tested in various experiment-like setups, but we focus here on modeling a 
purely passive cell to fully understand the mechanics at stake without migration or skeleton reorganization. In this regard, we first build a model of an isolated nucleus that will be tested under compression mimicking the experimental setup from Caille et al. [14]. This model being thoroughly investigated, it will be implemented in a whole cell model, modeling the cytoplasm as in previous work [33]. This complete model is then tested to reproduce a perfusion experiment [11]. The mechanical parameters of the cell and its nucleus were chosen to match those of the HeLa cell, as in our previous work [33], but the versatility of our model would allow it to cover a wide range of cell types by simply adjusting the mechanical parameters.

\section{Numerical simulation of a compression test of the nucleus}

\subsection{Nucleus geometry}

The nucleus $\left(\Omega_{\text {nucleus }}\right.$ ) has an initial circular geometry of radius $r_{\text {nucleus }}=4$ $\mu \mathrm{m}$ and is composed of the $50 \mathrm{~nm}$ thick lamina $\left(\Omega_{\text {lamina }}\right)$ and the nucleoplasm $\left(\Omega_{\text {nucleoplasm }}\right)$ (See Figure 1$)$. Both the lamina and the nucleoplasm have been described through characteristic functions $g_{l}$ and $g_{n p}$ which are a composition of a regularized Heaviside function and a level set function (see Appendices A and B). The characteristic function representing the whole nucleus is given by $g_{n}(\mathbf{x})=g_{n p}(\mathbf{x})+g_{l}(\mathbf{x})$, where $\mathbf{x}$ indicates the current position of any particle of the system.

\subsection{Constitutive model and mechanics of the nucleus}

In most models, the nucleus is simply described as a viscoelastic material. In order to tackle its potential irreversible deformation, we chose to implement a yisco-elasto-plastic material composed of the lamina, purely elastic, and the nucleoplasm, visco-elasto-plastic, as represented in the schema in Fig 2. Both nuclear components were assembled in parallel since we consider the stress from both components to add up. Indeed, the lamina being very thin, the associated mesh would need to be too small. The mechanical influence of the lamina is 


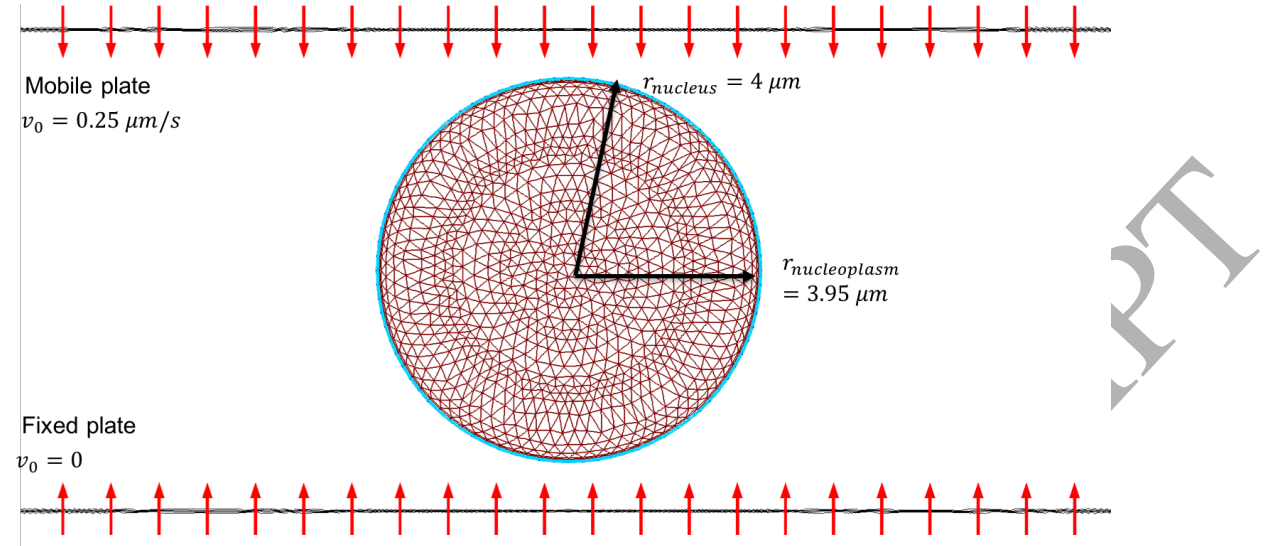

Figure 1: Geometry of the nucleus (Dark red: nucleoplasm and Light blue: lamina) and compression plates (red arrows represent the outward normal to the plates $\mathbf{n}_{\text {plate }}$ ) and FE mesh

thus not taken into account unless we homogenize the whole nucleus with volume ratios through the Voigt homogenization [39] (See Equation 4). An Arbitrary Lagrangian Eulerian (ALE) formulation is used in all the simulations, which allows to consider only small strains in the moving frame and Cauchy stresses $[40,41]$. The total Cauchy stress $\boldsymbol{\sigma}_{n}$, where the subscript $\mathrm{n}$ stands for nucleus, and the small deformation tensor $\boldsymbol{\epsilon}_{n}$ are defined as

$$
\begin{aligned}
& \boldsymbol{\sigma}_{n}=\left(\boldsymbol{\sigma}_{n p}+\boldsymbol{\sigma}_{l}\right) \\
& \boldsymbol{\epsilon}_{n}=\left(\frac{1}{2}\left(\mathbf{D}_{\mathbf{x}} \mathbf{u}+\mathbf{D}_{\mathbf{x}} \mathbf{u}^{T}\right)\right)=\boldsymbol{\epsilon}_{n p}=\boldsymbol{\epsilon}_{l}
\end{aligned}
$$

where the subscripts $n p$ and $l$ indicate the nucleoplasm and the lamina respectively, $\mathbf{u}$ is the displacement, $\mathbf{D}_{\mathbf{x}} \mathbf{u}$ is the usual displacement gradient.

The nucleoplasm itself is decomposed into a visco-plastic part and a purely elastic part as follows

$$
\begin{gathered}
\boldsymbol{\sigma}_{n p}=\boldsymbol{\sigma}_{n p, e}=\boldsymbol{\sigma}_{n p, v p} \\
\boldsymbol{\epsilon}_{n p}=\boldsymbol{\epsilon}_{n p, v p}+\boldsymbol{\epsilon}_{n p, e}
\end{gathered}
$$

where the subscripts $v p$ and $e$ stand for visco-plastic and elastic, respectively. 


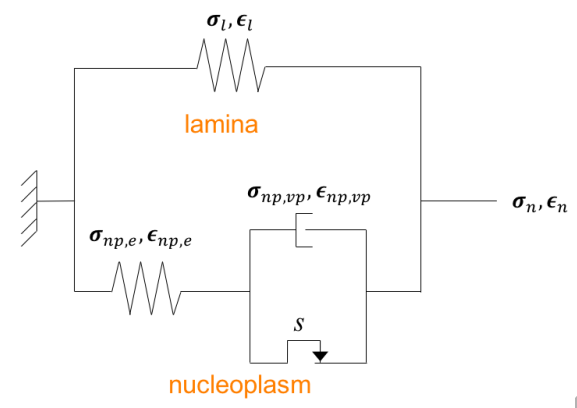

Figure 2: Rheological model of the homogenized nucleus

The constitutive equations of each part reads more specifically:

$$
\begin{aligned}
& \boldsymbol{\sigma}_{l}=\lambda_{l} \operatorname{Tr}\left(\boldsymbol{\epsilon}_{n}\right) \mathbf{I}+2 \mu_{l} \boldsymbol{\epsilon}_{n} \\
& \boldsymbol{\sigma}_{n p}=\lambda_{n p} \operatorname{Tr}\left(\boldsymbol{\epsilon}_{n}-\boldsymbol{\epsilon}_{n p, v p}\right) \mathbf{I}+2 \mu_{n p}\left(\boldsymbol{\epsilon}_{n}-\boldsymbol{\epsilon}_{n p, v p}\right) \\
& \dot{\boldsymbol{\epsilon}}_{n p, v p}^{D}=\frac{h\left(\boldsymbol{\sigma}_{n p, V M}-s\right)\left|\boldsymbol{\sigma}_{p, V M}-s\right|}{\eta_{n p}} \frac{\boldsymbol{\sigma}_{n p}^{D}}{\left\|\boldsymbol{\sigma}_{n p}^{D}\right\|}
\end{aligned}
$$

where $\lambda_{l}, \mu_{l}, \lambda_{n p}$ and $\mu_{n p}$ are the Lamé coefficients of the lamina and the nucleoplasm, respectively and defined as $\lambda_{k}=\frac{E_{k} \nu_{n}}{\left(1+\nu_{n}\right)\left(1-2 \nu_{n}\right)}$ and $\mu_{k}=\frac{E_{k}}{2\left(1+\nu_{n}\right)}$, $k=\{l, n p\} . E_{l}, E_{n p}$ and $\nu_{n}$ are the Young moduli of the lamina and the nucleoplasm respectively and the Poisson ratio of the nucleus. $\operatorname{Tr}$ defines the trace operator, $\boldsymbol{I}$ is the identity matrix and $\boldsymbol{A}^{D}$ indicates the deviatoric part of the tensor $\boldsymbol{A}$ defined as $\boldsymbol{A}^{D}=\boldsymbol{A}-\frac{1}{2} \operatorname{Tr}(\boldsymbol{A}) \mathbf{I}$. We hypothesize that $\boldsymbol{\epsilon}_{n p, v p}$ only has a deviatoric part. We note $h$ the regularized Heaviside function, $s$ the plasticity threshold, $\boldsymbol{\sigma}_{n p, V M}$ the Von Mises stress of the nucleoplasm and $\eta_{n p}$ the viscosity of the nucleoplasm defined as $\eta_{n p}=\tau_{n p} E_{n p}$, with $\tau_{n p}$ its characteristic time [35].

Due to the very small thickness of the lamina $(50 \mathrm{~nm})$, the Young moduli were weighted with regard to the surface occupied by the lamina and the 
nucleoplasm as:

$$
\begin{aligned}
E_{n p} & =E_{n p, 0} \frac{A_{n p, 0}}{A_{n, 0}} \\
E_{l} & =E_{l, 0} \frac{A_{l, 0}}{A_{n, 0}}
\end{aligned}
$$

with $A_{n p, 0}, A_{n, 0}$, and $A_{l, 0}$ being the initial areas of the nucleoplasm, the nucleus and the lamina, respectively.

The nominal values of the mechanical parameters $E_{l, 0}, E_{n p, 0}, \tau_{n p}$ and the Poisson ratio $\nu_{n}$ were taken from the literature and previous works. There is no experimental data allowing us to determine the value of the plasticity threshold $s$, so we performed various tests in order to find a consistent value. All parameters values and references are listed in Table 1.

\subsection{Compression experiment}

Initially, the nucleus is placed between two rigid plates $\left(\Omega_{\text {plate }}\right)$ of length $l_{\text {plate }}$ and height $g_{\text {plate }}$, the lower plate being fixed and the upper one being mobile, to simulate a compression experiment as in Caille et al. [14]. All parameters were chosen so that the compression speed is the same as in Caille et al. and the nucleus is compressed up to $70 \%$. Gravity is applied to the nucleus before the compression cycle begins. The upper $l_{u p}$ and lower $l_{l p}$ plates are described by two characteristic functions $g_{u p}(\mathbf{x})$ and $g_{l p}(\mathbf{x}-\mathbf{u})$, where $\mathbf{x}-\mathbf{u}$ indicates the initial position of any particle of the system (see Appendix C), in order to give the obstacles position to evaluate the contact force.

The friction force between the plate and the cell when they are in close contact is neglected. A normal force is introduced to control the contact between the nucleus and the plates, as follows

$$
\mathbf{f}_{\text {plate }}(\mathbf{x}-\mathbf{u})=\mu_{\text {plate }} g_{\text {plate }}(\mathbf{x}) \mathbf{n}_{\text {plate }}
$$

where $\mu_{\text {plate }}$ is the penalization coefficient, $\mathbf{u}$ is the displacement and $g_{\text {plate }}(\mathbf{x})=$ $g_{u p}(\mathbf{x})+g_{l p}(\mathbf{x})$ is a characteristic function that varies smoothly between 0 and 1 on a given interpenetration depth equal to $0.2 \mu \mathrm{m}$. This depth can be adjusted 
by varying the scaling parameter of the Heaviside function. Consequently, because of the property of the level set, no such force is applied when the plate and the nucleus are far from each other. When they become close enough, an eventual small overlap induces a large repulsive force cubically dependant of the overlap depth. Lastly, $\mathbf{n}_{\text {plate }}$ is the outward normal to the plates.

As described in previous work [33], the global equilibrium of the system is expressed as

$$
\rho_{n} \mathbf{a}=\operatorname{Div}\left(\boldsymbol{\sigma}_{n}\right)+\mathbf{f}_{\text {plate }}+\rho_{n} \mathbf{g}
$$

where $\rho_{n}$ is the nucleus density, $\mathbf{a}$ is the acceleration and Div is the usual divergence and $\mathbf{g}$ is the gravity acceleration. $\mathbf{f}_{\text {plate }}$ is the contact force, considered here as a localized body force in the neighbourhood of the contact with plate.

\subsection{Results}

The nucleus undergoes a loading/unloading cycle with $T_{1}, T_{2}$ and $T_{3}$ respectively being equal to $10,27.5$ and $2 \mathrm{~s}$. First, the nucleus settles down on the fixed lower plate under the action of gravity $\left(0<t<T_{1}\right)$. Then, the mobile upper plate goes down and compresses the nucleus up to $70 \%\left(T_{1}<t<T_{1}+T_{2}\right)$ and keeps the maximum compression for a time $T_{3}$. Lastly, the upper plate goes back to its initial position as it unloads the nucleus.

In a first simulation, we set $E_{l, 0}$ to $3000 \mathrm{~Pa}, E_{n p, 0}$ to $25 \mathrm{~Pa}, \tau_{n p}$ to $2 \mathrm{~s}$ and the plasticity threshold to $4 \mathrm{~Pa}$. Our model displays a non linear forcenormalized deformation ( with $d_{N}$ the normalized vertical deformation of the nucleus) relationship during compression that shows a similar profile than those in Caille et al. [14] (Figure 3a). Additionally, a plastic behaviour is observed since the norm of the average deviatoric strain $\epsilon_{n}^{D}$ reaches a peak of $70 \%$ at $\mathrm{t}=$ $30 \mathrm{~s}$ as expected, but does not drop back to zero once the nucleus is unloaded (between $\mathrm{t}=35 \mathrm{~s}$ and $\mathrm{t}=50 \mathrm{~s}$ ). In fact a residual strain of about $8 \%$ is found (Figure 3b). 

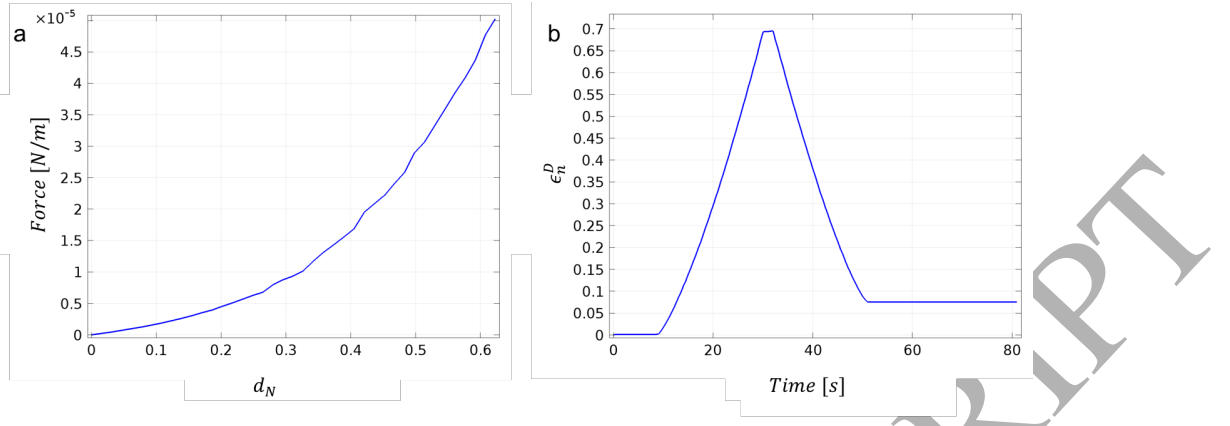

Figure 3: Simulation of compression and release of the nucleus. (a) Force-Normalized displacement curve, (b) evolution of the deviatoric strain $\epsilon_{n}^{D}$ in the nucleus with respect to time

Four test cases were implemented to study the influence of several parameters on the behaviour of the model. First, we examined the response of the nucleus model for $E_{l, 0}$ equal to 100, 500, 1000, 3000 and $10000 \mathrm{~Pa}$ (Figure 4). When the lamina's Young modulus increases, the forceneeded to compress the nucleus increases as well (Figure 4a), while the plastic deviatoric strain after unloading decreases (Figure 4b). To compress the nucleus up to $50 \%$, the force ranges from $6 \mu \mathrm{N} / \mathrm{m}$ for $E_{l, 0}=100 \mathrm{~Pa}$ to $80 \mu \mathrm{N} / \mathrm{m}$ for $E_{l, 0}=10000 \mathrm{~Pa}$, while the plastic deviatoric strain after unloading ranges from $30 \%$ to $2.5 \%$ (Figure $4 \mathrm{a}$ ).
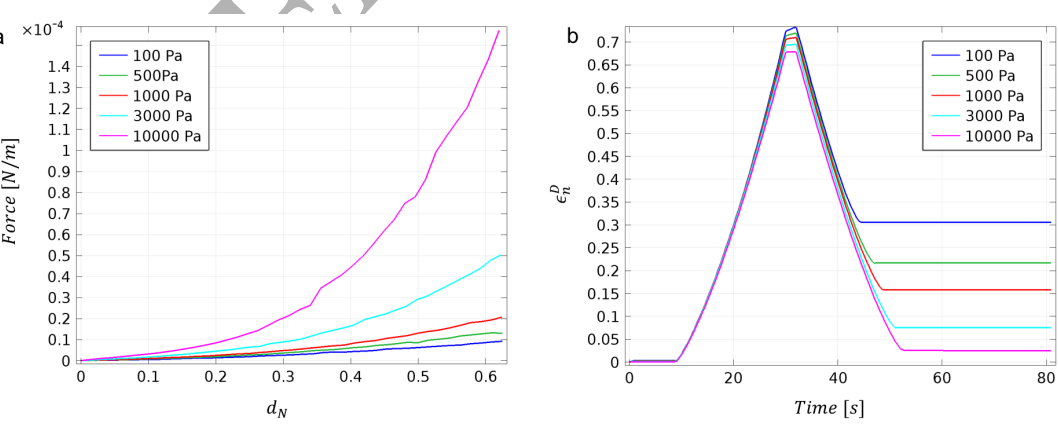

Figure 4: (a) Force-Normalized displacement curve and (b) evolution of the nuclear deviatoric strain $\boldsymbol{\epsilon}_{n}^{D}$ with respect to time for various values of $E_{\text {lamina,0 }}$

Thus, the lamina seems to be a major load-bearing element of the nucleus 
and a lower Young modulus, which can be correlated with lamin-deficient nuclei, triggers higher nuclear plasticity. This result is consistent with a recent study on nuclear deformability [37] in which irreversible nuclear deformation was observed after transmigration of Lamin $\mathrm{A} / \mathrm{C}$ deficient cells.

Although most reviews focus on the lamina [42, 43], the recent work of Stephens et al. highlighted the fact that chromatin may play a critical role as well [30]. The influence of the stiffness of the nucleoplasm, in which the chromatin has a major impact, was then considered with $E_{n p, 0}$ equal to 1 , 25, 100 and $1000 \mathrm{~Pa}$ (Figure 5). As expected, the force required to/compress the nucleus increased with the Young modulus (Figure 5a). Contrarily to the previous case, a higher nucleoplasm stiffness yielded to an increased plasticity (Figure 5b). The deviatoric strain upon unloading of the mucleus goes down at various speeds due to the definition of $\eta_{n p}=\tau_{n p} E_{n p}$. Here, the force required for a $50 \%$ compression ranges from 20 to $100 \mu N / m$ (see Figure 5a). Thus, as well as the lamina, our model features the nucleoplasm as a potential load-bearing element.
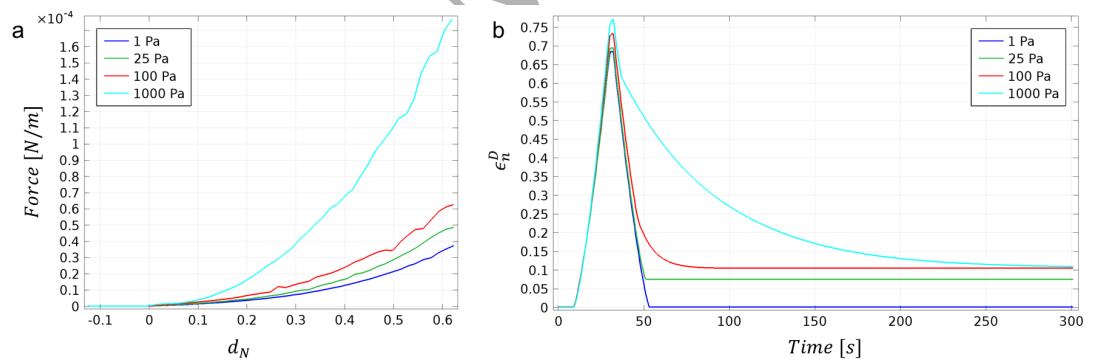

Figure 5: (a) Force-Normalized displacement curve and (b) evolution of the nuclear deviatoric strain with respect to time for various values of $E_{n u c l e o p l a s m, 0}$

Finally, $\tau_{n p}$ and $s$ could also affect the overall mechanical behaviour of the nucleus. The nucleoplasm characteristic time has been found to be very disperse depending on the cell and experiment type. In this simulation, $\tau_{n p}$ was set to $0.1,1,2,10$ and $30 \mathrm{~s}$ (Figure 6b). Longer characteristic times gave less plastic 
behaviours down to only $2 \%$. As for the influence of $s$, with values of $0.1,1$, 2, 4, 10 and $30 \mathrm{~Pa}$ (Figure 6a), the last two values yielded roughly no plastic strain. It is interesting to notice that there is an increased plasticity from 0 to $5 \%$ when the threshold increases from 0.1 to $4 \mathrm{~Pa}$ (see Figure 6).
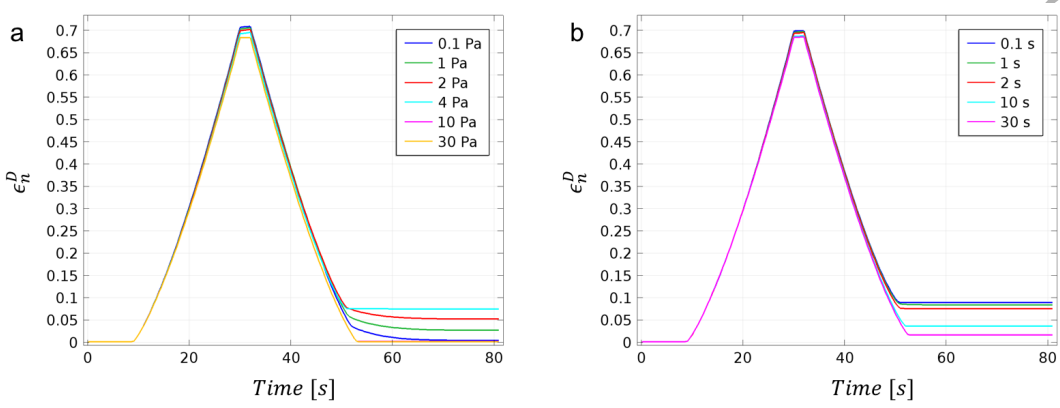

Figure 6: Deviatoric strain for various values of plasticity threshold (a) and $\tau_{\text {nucleoplasm }}(\mathrm{b})$

\subsection{Nuclear plasticity correlates with the lamin levels and nucleoplasm stiffness}

In conclusion, our model does reproduce the visco-elastic behaviour of the cell's nucleus, as well as its plasticity upon higher strains. Depending on the values of Young's moduli of the lamina and the nucleoplasm, either of them can be the major load-bearing element of the nucleus. With respect to the experimental works in the literature, we chose to set the lamina as the major load-bearing element and the nucleoplasm as the one prone to plasticity. The aforementioned plasticity grows larger with increasing values of $E_{n p, 0}$ and with decreasing values of $E_{l, 0}$ or $\tau_{n p}$. Smaller value of $E_{l, 0}$ can be seen as the modeling equivalent of lamin-deficient nuclei, hence being in agreement with experimental results where Lamin A/C-deficient nuclei present a larger plastic deformation [37]. Now that the compression study has been completed, we aim to implement the nucleus constitutive law into a whole cellular model to simulate a perfusion test. 


\section{2D model of a perfusion experiment}

In this section we detail the 2D FE model developed to simulate a perfusion test. In this type of experiment, the cell passively flows through micro-channels in which a fluid flow is assured thanks to a pressure gradient [11]. Cell deformability and passing time can be studied. The objective here is to analyze the influence of the nucleus visco-elasto-plastic behavior during perfusion on the overall cellular response. Such influence will be studied more specifically on two phenotypes through variation of the mechanical properties of the lamina: a control model and a lamin-deficient model of the cell.

\subsection{Cell geometry}

Similarly to our previous works $[33,44]$, the cell $\left(\Omega_{\text {cell }}\right)$ is constituted by the surrounding actin cortex $\left(\Omega_{\text {cortex }}\right)$ and the cytosol $\left(\Omega_{\text {cytosol }}\right)$, which form the cytoplasm $\left(\Omega_{\text {cytoplasm }}\right)$, and by the lamina $\left(\Omega_{\text {lamina }}\right.$, Sec. 2.2$)$ and the nucleoplasm $\left(\Omega_{\text {nucleoplasm }}\right.$, Sec. 2.2$)$, which form the nucleus $\left(\Omega_{\text {nucleus }}\right.$, Sec. 2.2) (Figure 7). To describe the $\Omega_{\text {cortex }}$ and the $\Omega_{\text {cytosol }}$, we use, as we did for the lamina and the nucleoplasm (Sec, 2.1) two characteristic functions $g_{c x}(\mathbf{x})$ and $g_{c l}(\mathbf{x})$ as described in the Appendix D (Figure 7).

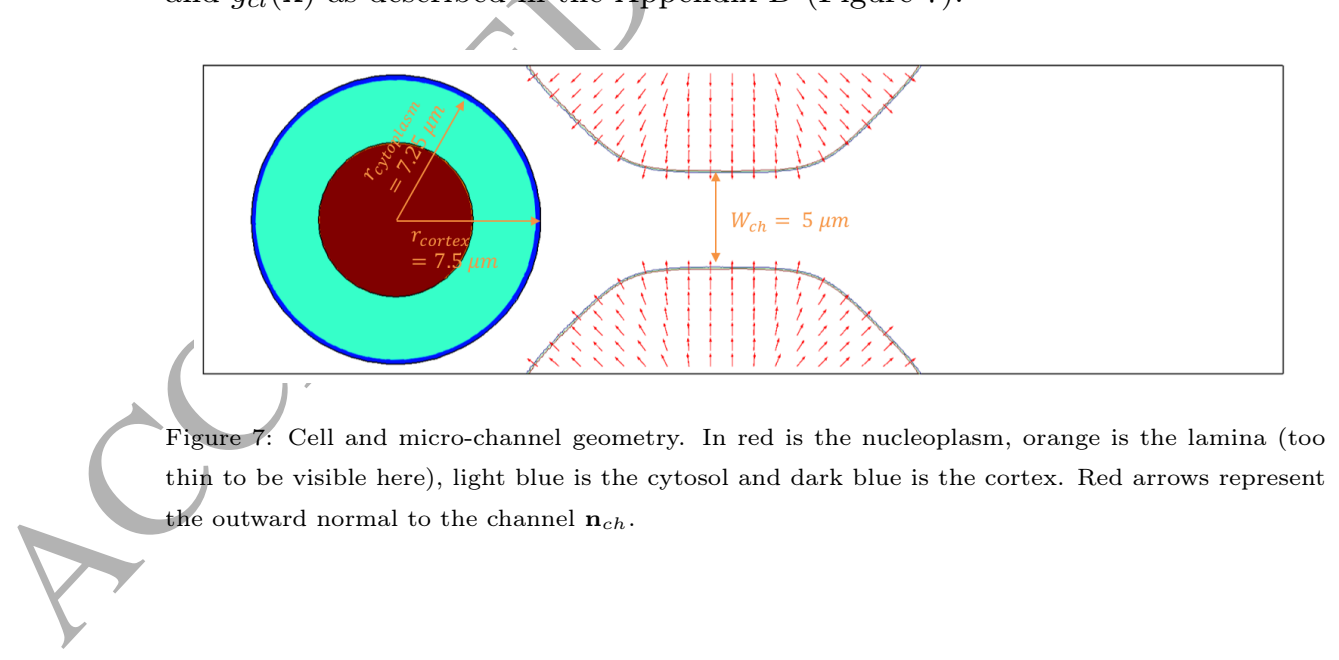




\subsection{Perfusion experiment}

The perfusion device is modeled as a constriction channel $\left(\Omega_{\text {channel }}\right)$ defined by two rigid walls. The upper $\left(g_{u c}\right)$ and the lower $\left(g_{l c}\right)$ walls (Figure 7 ) are defined by two characteristic functions (Appendix E).

We first performe a microfluidic study to obtain the velocity field inside the micro-channel. The fluid is described by the Navier-Stokes equation as follows

$$
\rho_{f} \boldsymbol{a}_{f}=-\nabla p \mathbf{I}+\eta_{f} \Delta \boldsymbol{v}_{f}
$$

where $\rho_{f}$ is the volumetric mass of the fluid, $\boldsymbol{a}_{f}$ the fluid acceleration, $\nabla$ the gradient operator, $p$ the pressure, $\eta_{f}$ the fluid viscosity, $\Delta$ the Laplacian and $\boldsymbol{v}_{f}$ the fluid velocity.

The boundary conditions include a pressure $P_{\text {in }}$ at the left inlet and $P_{\text {out }}$ at the right outlet. The fluid parameters are chosen in order to avoid turbulent patterns both at the entrance and the exit of the constriction (Figure 8 and Table 1). Finally, we assume that the velocity field calculated without the cell in the constriction does not vary over the perfusion assay, which constitutes a first step towards a more complex multiphysics model including fluid-structure interactions that will not be developed here.

The force $\mathbf{f}_{\text {fluid }}$ exerted by the fluid on the cell cytoplasm is then defined as

$$
\mathbf{f}_{\text {fluid }}(\mathbf{x})=f_{\text {fluid }, 0} \boldsymbol{v}_{f}
$$

with $f_{\text {fluid,0 }}$ the amplitude of the force applied on the cell, chosen so that the cell always passes through the channel in $1 s \pm 0.05 s$ in order to be able to compare the results (experimentally, the passing time in such devices ranges from $20 \mathrm{~ms}$ to a few seconds $[45,19,20])$.

Similarly to the compression test, a contact force is applied, now using the regularized characteristic function of the channel, as follows

$$
\mathbf{f}_{c h}(\mathbf{x})=\mu_{c h} g_{c h}(\mathbf{x}) \mathbf{n}_{c h}
$$




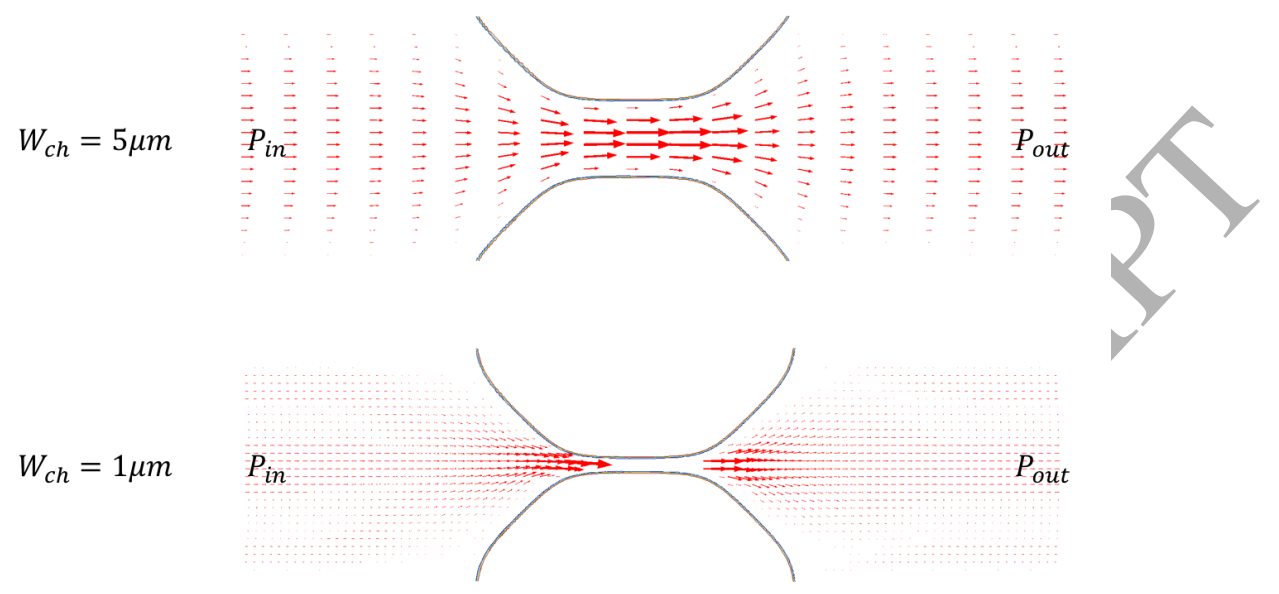

Figure 8: Profile of the fluid velocity inside the device for two sizes of constriction $W_{c h}=5$ and 1 $\mu m$

where $\mu_{c h}$ is the penalization coefficient, $g_{c h}=g_{u c}+g_{l c}$ is a characteristic function as defined for $g_{\text {plate }}$ in Section 2.3 and $\mathbf{n}_{c h}$ is the outward normal to the channel.

\subsection{Constitutive model and mechanics of the cytoplasm}

As the nucleus constitutive law has been presented in Sec. 2.2, here we describe the mechanicalbehaviour of the other cell's components, namely the actin cortex and the cytosol. We assume that the cortex behaves as an isotropic elastic material, whereas the cytosol is described as viscoelastic, the whole cytoplasm thus being described by a generalized Maxwell model [44, 46, 33] (Figure 9). As previously developed, the cytoplasm is considered as a Voigt-homogenized material from its two constituents, namely the cortex and the cytosol. The whole cell is then a heterogeneous composed of the homogenized cytoplasm and the homogenized nucleus, such that $\boldsymbol{\sigma}=g_{n} \boldsymbol{\sigma}_{n}+g_{c p} \boldsymbol{\sigma}_{c p}$. The boundary conditions between these components are handled by the level set functions and the finite 
elements. The Cauchy stress of the cytoplasm $\boldsymbol{\sigma}_{c p}$ is given by

$$
\boldsymbol{\sigma}_{c p}=g_{c p}(\mathbf{x})\left(\boldsymbol{\sigma}_{c x}+\boldsymbol{\sigma}_{c l}\right)
$$

where the subscripts $c p, c x$ and $c l$ represent the cytoplasm, the cortex and the cytosol respectively, and $g_{c p}$ represents the characteristic function of the cytoplasm (see Appendix D).

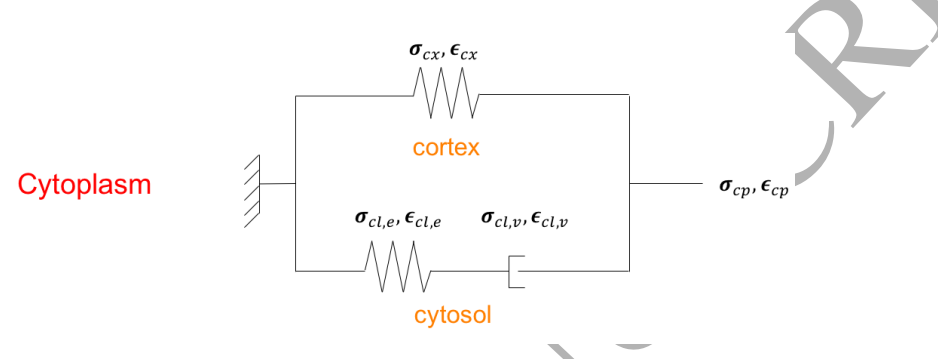

Figure 9: Rheological model of the cytoplasm [33]

The cytosol itself is decomposed into a viscous part and a purely elastic part as follows

$$
\begin{gathered}
\sigma_{c l}=\sigma_{c l, e}=\sigma_{c l, v} \\
\epsilon_{c l}=\boldsymbol{\epsilon}_{c l, v}+\boldsymbol{\epsilon}_{c l, e}
\end{gathered}
$$

where the subscripts $v$ and $e$ respectively stand for viscous and elastic.

Additionally, we have

$$
\begin{aligned}
& \boldsymbol{\sigma}_{c x}=\lambda_{c x} \operatorname{Tr}\left(\boldsymbol{\epsilon}_{c p}\right) \mathbf{I}+2 \mu_{c x} \boldsymbol{\epsilon}_{c p} \\
& \boldsymbol{\sigma}_{c l}=\lambda_{c l} \operatorname{Tr}\left(\boldsymbol{\epsilon}_{c p}-\boldsymbol{\epsilon}_{c l, v}\right) \mathbf{I}+2 \mu_{n p}\left(\boldsymbol{\epsilon}_{c p}-\boldsymbol{\epsilon}_{c l, v}\right) \\
& \dot{\boldsymbol{\epsilon}}_{c l, v}^{D}=\frac{\boldsymbol{\sigma}_{c l}^{D}}{\eta_{c l}}
\end{aligned}
$$

where $\lambda_{c x}, \mu_{c x}, \lambda_{c l}$ and $\mu_{c l}$ are the Lamé coefficients associated to the cortex and the cytosol respectively, defined as $\lambda_{k}=\frac{E_{k} \nu_{c}}{\left(1+\nu_{c}\right)\left(1-2 \nu_{c}\right)}$ and $\mu_{k}=\frac{E_{k}}{2\left(1+\nu_{c}\right)}$, $k=\{c x, c l\} . E_{c x}, E_{c l}$ and $\nu_{c}$ are the Young moduli of the cortex and the cytosol respectively and the Poisson ratio of the cytoplasm. $\eta_{c l}$ is the viscosity of the 
cytosol defined as $\eta_{c l}=\tau_{c l} E_{c l}$, with $\tau_{c l}$ its characteristic time [35]. As before, we assume that $\boldsymbol{\epsilon}_{c l, v}$ only has a deviatoric part.

As previously, and due to the very small thickness of the cortex $(10 \mathrm{~nm})$, the Young moduli were homogenized with regard to the surface occupied by the cortex and the cytosol as:

$$
\begin{aligned}
E_{c x} & =E_{c x, 0} \frac{A_{c x, 0}}{A_{c p, 0}} \\
E_{c l} & =E_{c l, 0} \frac{A_{c l, 0}}{A_{c p, 0}}
\end{aligned}
$$

where $A_{c x, 0}, A_{c p, 0}$, and $A_{c l, 0}$ are the initial areas taken respectively by the cortex, the cytoplasm and the cytosol.

The nominal values of the mechanical parameters $E_{c x, 0}, E_{c l, 0}, \tau_{c l}$ and the Poisson ratio $\nu_{c p}$ were taken from the literature and previous work as listed in Table 1.

Similarly to the nucleus computation, an ALE formulation with updated frame and moving mesh is adopted. The contact force is also considered as a body force only applied in the possible overlapped region between the cell and the channel whenever it takes place and measured by the intersection level set of the channel and the cell and the global equilibrium of the system is expressed as

$$
\rho \mathbf{a}=\operatorname{Div}(\boldsymbol{\sigma})+\mathbf{f}_{\text {fluid }}+\mathbf{f}_{c h}
$$

where $\rho$ is the cell density defined as $\rho(\mathbf{x})=\rho_{n} g_{n}(\mathbf{x})+\rho_{c p} g_{c p}(\mathbf{x})$, a is the acceleration and $\boldsymbol{\sigma}$ is the Cauchy stress that reads $\boldsymbol{\sigma}=g_{n} \boldsymbol{\sigma}_{n}+g_{c p} \boldsymbol{\sigma}_{c p}$. $\mathbf{f}_{\text {fluid }}$ and $\mathbf{f}_{c h}$ respectively indicate the fluid force exerted on the cell cytoplasm and the contact force between the cell and the channel.

\subsection{Results}

In this study, we tackle two channel widths: $W_{c h}=5 \mu m$ (slightly subnuclear) and $W_{c h}=1 \mu m$ (highly sub-nuclear). The large channel corresponds to a common size for perfusion experiment [11], while the 1-micron channel was 
chosen to study the behaviour of the cell under higher constriction at a size close to the $10 \%$ limit impeding cell migration completely [7]. The fluid force applied to the cell is designed so that the passing time through the constriction is of the order of $1 \mathrm{~s}$, seconds, as can be found experimentally [45, 19, 20]. In each case, we are first interested in the displacement of the cell inertia center $\mathbf{d}_{c}$ that decomposes as $d_{c, x}$ and $d_{c, y}$ on both directions with $d_{c, y}$ equal to 0 . Then we study the evolution of the norm of the nucleus deviatoric strain $\epsilon_{n}^{D}$, the value and distribution of the Von Mises stress, the ratio of nucleus area over cell area $\frac{A_{n u c}}{A_{\text {cell }}}$ and the vertical and horizontal component of the positive part of the resultant of the fluid force acting on the cell $\left(\left|F_{\text {fluid, } x}\right|\right.$ and $\left|F_{\text {fluid,y }}\right|$ respectively), all with respect to $d_{c, x}$. To better understand the structural role of the lamina and the chromatin, we implement one model for a "wild-type" cell and another for a "lamin-deficient" one, in which $E_{l, 0}$ is set to 3000 and 30 $\mathrm{Pa}$, respectively.

\subsubsection{5 microns-wide constriction}

We first look at the "wild-type" model in the larger channel. The cell rapidly plugs the channel $\left(d_{c, x}=4 \mu m\right)$ and then requires more time until the nucleus itself clogs the channel $\left(d_{c, x}=12 \mu m\right)$. Once this is accomplished, the cell goes through very rapidly (it takes here 1-2 ms, which is consistent with the results presented in [11]) and can then go back to a relaxed state (Figure 10 and Movie 1 in Supplementary Material).

The horizontal displacement of the cell center of inertia $d_{c, x}$ illustrates this phenomenon clearly (Figure 11.a). The deviatoric strain of the nucleus reaches a maximum of $35 \%$ at the center of the constriction and no plastic deformation is found in the nucleus (Figure 11.b). The Von Mises stress reaches a maximum of $5.39 \mathrm{~Pa}$ in the constriction, which is barely above the plasticity threshold, thus explaining the absence of plasticity (Movie 1 in Supplementary Material). Initially, the cell and the nucleus area are equal to $176 \mu \mathrm{m}^{2}$ and $50 \mu \mathrm{m}^{2}$ respectively. Thus, the ratio of the nucleus over the cell area thus is $28.5 \%$ before entering the constriction. As expected, this ratio grows larger as the cell enters 


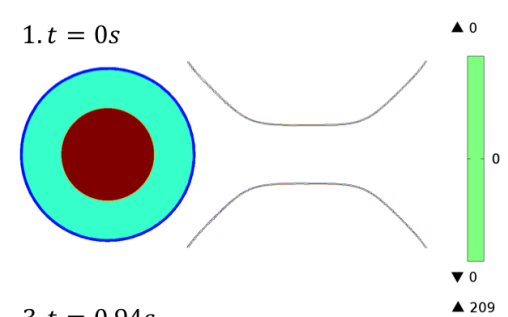

2. $t=0.1 s$
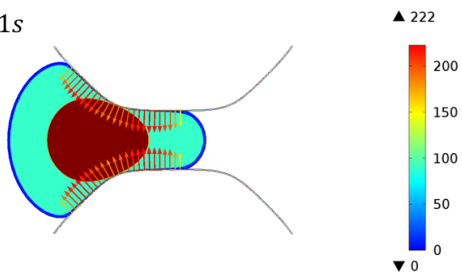

3. $t=0.94 s$

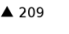

4. $t=0.9728 s$
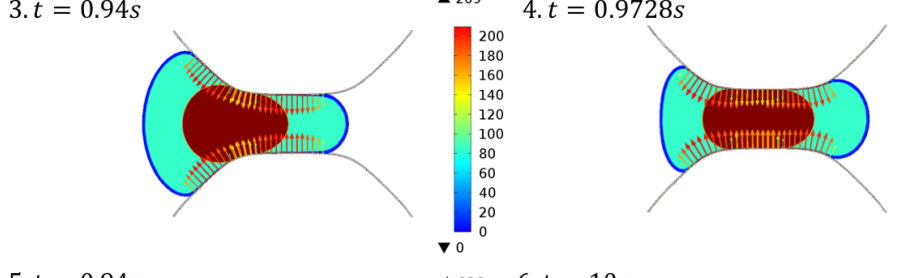

$\Delta 225$

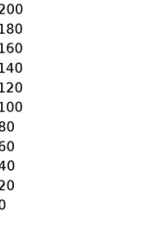

5. $t=0.94 s$

$\Delta 236 \quad$ 6.t $=10 \mathrm{~s}$
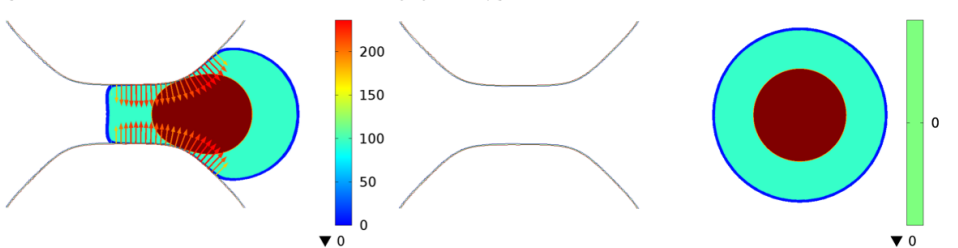

Figure 10: Simulation of the perfusion test in the $5 \mu m$-wide micro-channel for a wild-type cell. Arrows represent the norm of the contact force (refer to the color scale for the value)

the constriction to reach a peak of $40 \%$ for $d_{c, x}=16 \mu \mathrm{m}$ and it goes back to its initial value once the cell is completely out of the constriction after a displacement of $30 \mu \mathrm{m}$ (Figure 11.c)). The resulting force applied on the fluid reaches 80 and $17 \mathrm{pN}$ in the vertical and horizontal direction respectively (Figure 11.d). If the absolute value of the force is not specifically relevant by itself, it will be interesting to compare these value with the other test cases.

In the lamin-deficient model, the displacement of the cell inertia follows a similar curve, but the other parameters present significant differences (Figure 11). $\epsilon_{n u c}^{D}$ goes up to $62 \%$, which means a $77 \%$ higher nucleus compression than for the wild-type model. The Von Mises stress reaches a maximum of 7.3 $\mathrm{Pa}$ in the constriction, which is slightly higher than before and should yield a higher plasticity not observed here (Movie 2 in Supplementary Material). This seemingly contradictory result will be discussed later in this section. The area 

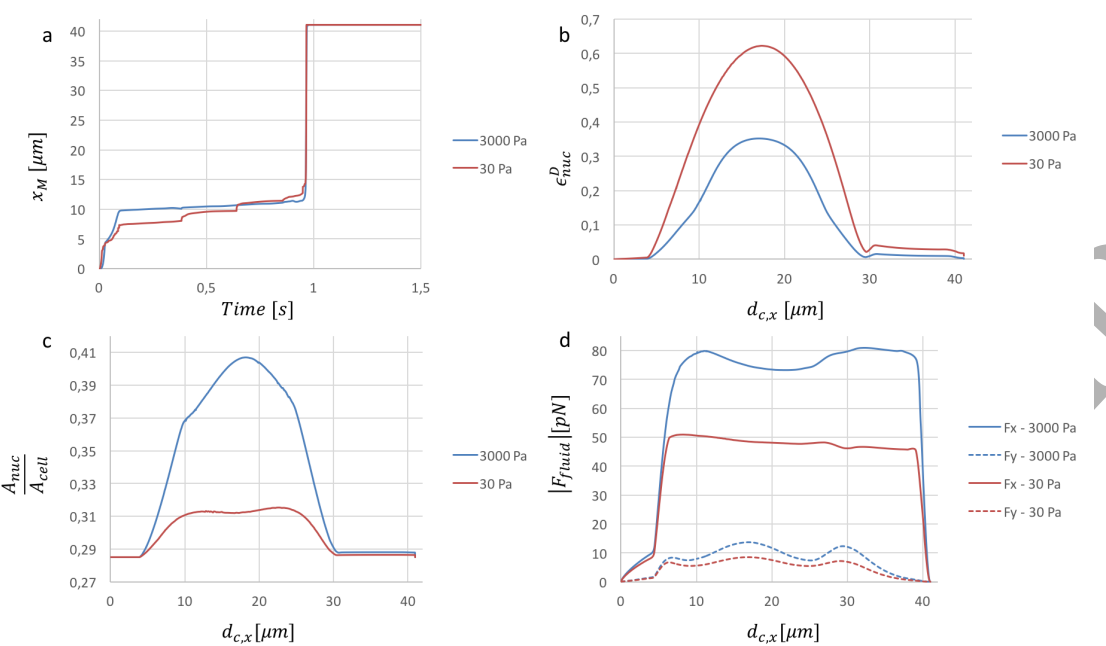

Figure 11: Comparison of the perfusion test results in the $5 \mu \mathrm{m}$-wide micro-channel for the wildtype (blue: $E_{l, 0}=3000 \mathrm{~Pa}$ ) and the lamin-deficient (red: $E_{l, 0}=30 \mathrm{~Pa}$ ) model. a) Horizontal displacement of the cell inertia center $d_{c, x}$, b) Nucleus deviatoric strain, c) Nucleus area relative to whole cell area and d) Resulting fluid force (vertical and horizontal components)

ratio curve shows two local maxima of 31 and $31.5 \%$ for $d_{c, x}=12$ and $24 \mu \mathrm{m}$. Both values are around $20 \%$ lower than previously, which reinforces the earlier finding that the nucleus undergoes more deformation when the lamina does not "shield" it from stress. As it could be expected, the force required to get the nucleus through the constriction is lower now that the lamina is depleted as it reaches 51 and $9 \mathrm{pN}$ in the vertical and horizontal direction respectively.

\subsubsection{1 micron-wide constriction}

With the strongly sub-nuclear channel and the wild-type model, we could have expected a similar behaviour as previously, only slower. However, the cell undergoes a different path to go through the smaller constriction. Indeed, as previously, the cell rapidly clogs the constriction $\left(d_{c, x}=4 \mu m\right)$, but then, the nucleus rapidly clogs the constriction $\left(d_{c, x}=12 \mu \mathrm{m}\right)$. Eventually, the nucleus gets gradually squeezed inside the constriction until the cell center of inertia displacement reaches $14 \mu \mathrm{m}$, where the cell finally goes through at once (Figures 
12 and 13a. and Movie 3 in Supplementary Material).

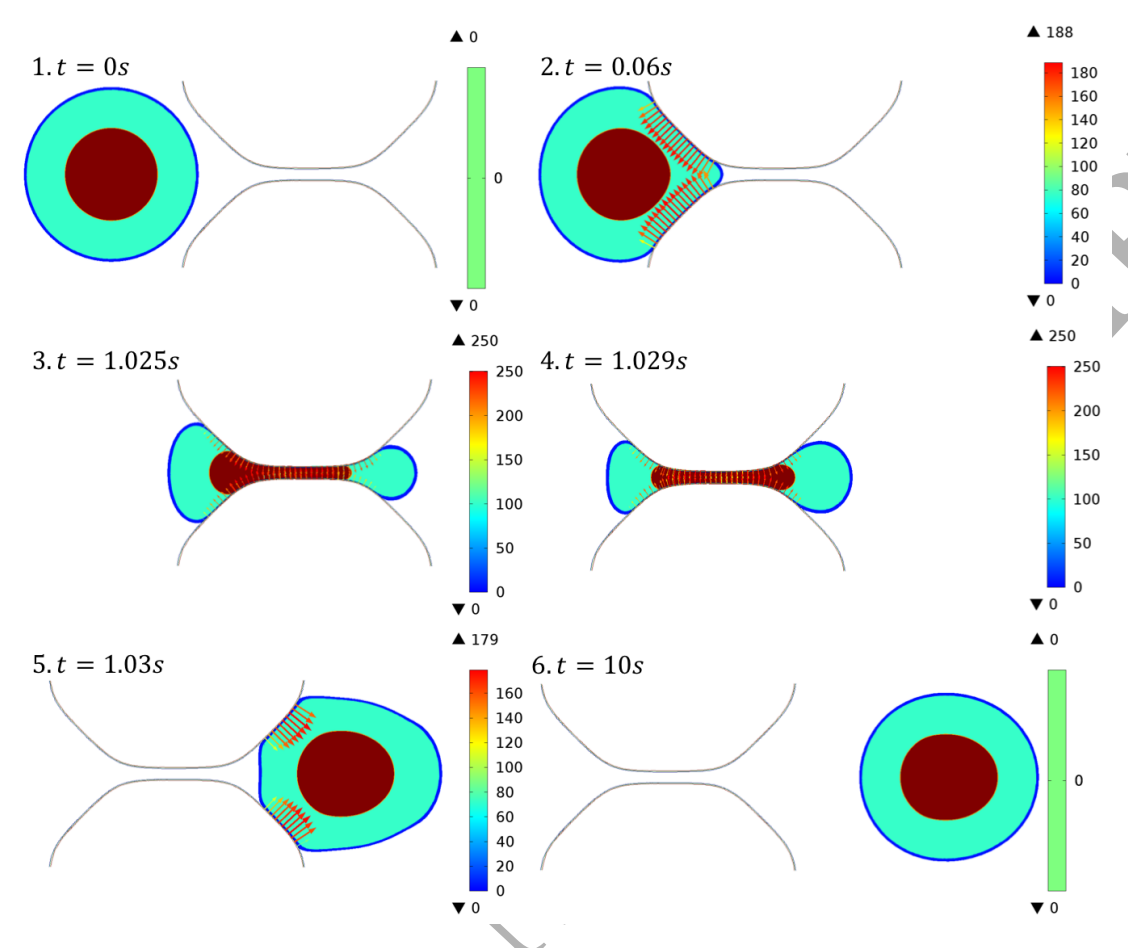

Figure 12: Simulation of the perfusion test in the $1 \mu \mathrm{m}$-wide micro-channel for a wild-type cell. Arrows represent the norm of the contact force (refer to the color scale for the value)

In this test case, the average nucleus deviatoric strain reaches $88 \%$ at $d_{c, x}=$ $18 \mu m$ (Figure 13b.), which corresponds to the time when the rear of the nucleus is fully inside the constriction. Here, the nucleus presents a plastic strain of $8 \%$ after reaching the other side of the constriction. The Von Mises stress reaches a maximum of $13.7 \mathrm{~Pa}$ at the center of the constrained the nucleus as it first clogs the channel and then displays a relaxation (Movie 3 in Supplementary Material). Looking at the ratio of the nucleus area over the cell area, the behaviour is significantly different from the $5 \mu \mathrm{m}$-wide channel since two maxima are clearly visible (Figure 13c.). The ratio reaches a maximum of $38 \%$ at $d_{c, x}=6.5 \mu \mathrm{m}$, corresponding to the nucleus starting to plug the channel, the front of the cytosol thus being already squeezed inside the constriction. The ratio then decreases as 
the nucleus makes its way through the constriction and there is a second peak at $40 \%$ for $d_{c, x}=28 \mu \mathrm{m}$ as the rear of the nucleus exits the constriction, before it settles down to $28.4 \%$ at the end of the perfusion test (Figure 13c.). The force needed for the cell to go through the constriction reaches 120 and $22 \mathrm{pN}$ in the $\mathrm{x}$ and $\mathrm{y}$ direction respectively (Figure 13d.), which is $50 \%$ higher, for the vertical component, as in the $5-\mu m$ channel.
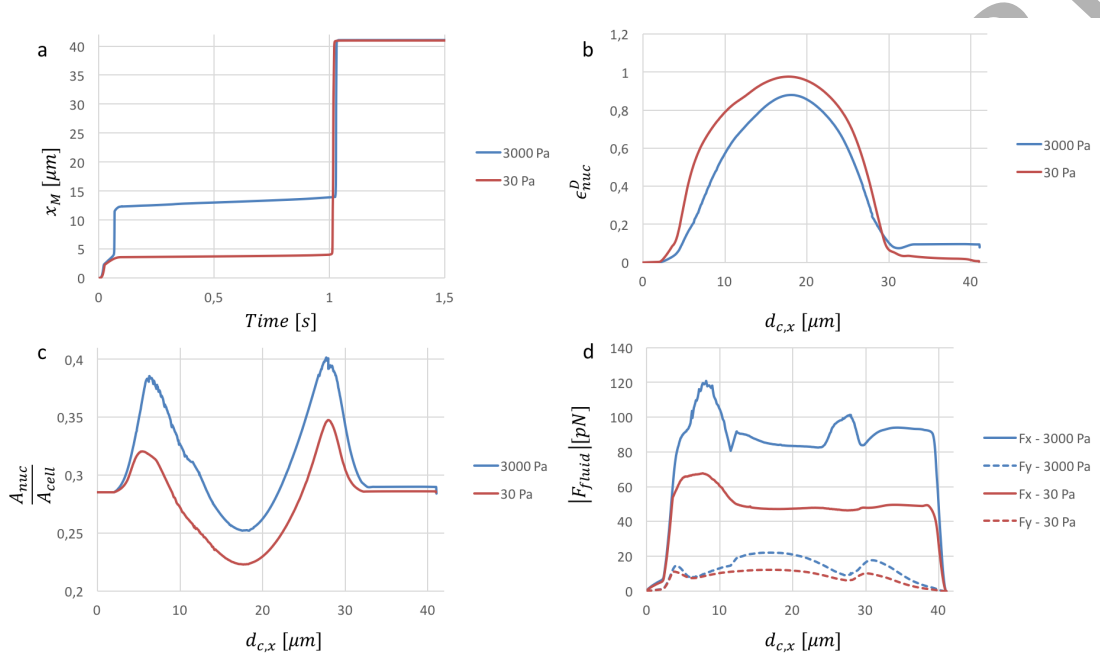

Figure 13: Comparison of the perfusion test results in the $1 \mu m$-wide micro-channel for wild type and lamin deficient cells. a) Horizontal displacement of the cell inertia center $d_{c, x}$, b) Nucleus deviatoric strain, c) Nucleus area relative to whole cell area and d) Resulting fluid force (vertical and horizontal components)

As for the lamin-deficient model, starting in the cell inertia center displacement, differences can be spotted from the wild-type case (Figure 13). The cell first plugs the channel $\left(d_{c, x}=3.5 \mu m\right)$ and, as soon as the cytoplasm tip starts getting into the constriction $\left(d_{c, x}=4 \mu m\right)$, the whole cell goes through. Thus, the nucleus itself does not seem to be a barrier to transmigration anymore. The deviatoric strain reaches $98 \%$, which is $10 \%$ higher than for the wild-type model. However, there is no plastic strain anymore compared to the wild-type, which is in contradiction with experimental results from Cao et al. [37]. This result 
will be further discussed in the following section. The Von Mises stress reaches a maximum of $16.2 \mathrm{~Pa}$ at the center of the constrained part of the nucleus as it starts clogging the constriction and then displays a relaxation (Movie 4 in Supplementary Material). Again, this value, superior than in the wild-type cell should result in an increase of the plasticity. The area ratio between the nucleus and the cell follows the same pattern than previously although with lower values: it reaches a first maximum of $32 \%$ for $d_{c, x}=5.5 \mu \mathrm{m}$ and second one of $35 \%$ for $d_{c, x}=28 \mu \mathrm{m}$. Together with the values of deviatoric strain, this shows, as in the larger channel, that the nucleus gets more squeezed if no lamina protects it. The force of the fluid on the cell reaches 67 and $12 \mathrm{pN}$ for its vertical and horizontal component respectively, which is almost half the force needed for the wild-type cell, once again comforting us in the idea that the nucleus is a major barrier during transmigration through small constrictions, and the lamina seems to be a crucial structural constituent of the nucleus allowing it to resist to large deformation. The decrease of plastic strain in the lamin-deficient model however raises the question of the role of the nucleoplasm as a load-bearing element of the nucleus.

\subsection{The cytoplasm can "pull" on a too soft nucleus}

The complete cell model with a visco-elasto-plastic nucleus was tested in 5 $\mu m$-wide and a $1 \mu m$-wide perfusion channel both as a wild-type cell $\left(E_{l, 0}=\right.$ $3000 \mathrm{~Pa}$ ) and a lamin-deficient one in which the lamina was considered a hundred times weaker $\left(E_{l, 0}=30 \mathrm{~Pa}\right)$. In both cases, the cell first plugs the device and then goes through very rapidly once it has progressed far enough in the constriction with a passage time of the order of $1 \mathrm{~ms}$. In the larger channel, once the nucleus is in contact with both the upper and lower channels, it is sufficiently compressed for the cell to go through directly. However, in the smaller constriction, an additional step is necessary for the nucleus to fully plug the channel and only once the cell center of inertia has progressed far enough in the constriction, the whole cell can pass through.

The study of the lamin-deficient model gives very interesting results and 
insights of the importance of the interaction between the cytoplasm and the nucleus. First, the lamin-deficient nucleus undergoes significantly higher deformation than the wild-type one, hence showing the importance of the lamina as a load-bearing component of the nucleus. This seems to back up studies stating that the lamina is the major structural constituent in the nucleus. However, a closer look on the behaviour of the nucleus with regards to plasticity shows that the wild-type model displays a $10 \%$ plastic strain after exiting the smaller constriction, while the lamin-deficient one does not display any irreversible deformation (a similar but less intense phenomenon is obseryed in the larger constriction regarding the Von Mises stress). This property of our model appears in contrast with the latest experimental results as diseussed by Cao et al. [37]. Further analysis of our results revealed that the cell cytoplasm seems responsible for this loss of plasticity as it forces the lamin-deficient soft nucleus to return to its original shape. This is an exciting result that meets the recent thesis defended by Stephens et al. [30] that not only does the lamina play a role but chromatin also plays a major part as a load-bearing element in the nucleus.

\section{Conclusion and Discussion}

All in all, our model of an isolated nucleus does display a plastic behaviour under extensive compression, as shown experimentally [28], and shows that both the lamina and the nucleoplasm have an impact on the mechanical behaviour of the overall nucleus. A softer lamina and a stiffer nucleoplasm yield a higher irreversible deformation after unloading the nucleus. Implementing this model in a whole cell model under a perfusion test allowed us to unveil the mechanical influence of the cytoplasm on the nucleus. In the $1 \mu \mathrm{m}$ constriction, the plastic deformation significantly decreased with the depletion of the lamina, which we explain by the cytoplasm "pulling" on the now softer nucleus to get it back to its original shape. This is further confirmed by the fact that in the isolated nucleus model, a decreased lamina Young modulus resulted in an increased plasticity. Thus, in order to observe plastic deformation, either the nucleoplasm should 
be stiffer, or the cytoplasm softer. This mechanical interplay between nuclear stiffness and its plasticity reveals the ability of numerical simulation to tackle biological issues and to shed light on different mechanisms.

Our model however presents some limitations and some improvements can be included. First of all, a 2D FE model was used, whereas a three-dimensional (3D) one would be needed in order to faithfully reproduce 3D experiments. This point is particularly sensitive looking at the compression test: a more valid reduction would be an axi-symmetric model. We still chose to develop a purely 2D model since it fits the perfusion test: the compression test model is a step towards a more complete cell model and a $2 \mathrm{D}$ reduction will not give faithful quantitative results, but the qualitative trends stay valid.

Moreover, we chose to cope with the thinness of the cortex and the lamina through weighted Young's moduli with respect to the relative area of each cell component. Nevertheless, a shell modél would surely fit better and could provide new insights on the relation between the various cell components [47].

Lately, some very interesting models of the cell nucleus have been developed $[32,37]$ in which the interaction between the nucleus and the cytoplasm is represented by an active force puling on the nucleus. Our model gives a new insight on the passive interaction between these 2 cellular compartments: the comparison between the isolated nucleus model and the complete cell model proves the major mechanical influence the cytoplasm can have on the nucleus in a passive state. This interaction would be increasingly important during an active phenomenon like transmigration.

\section{Acknowledgement}

This work was funded by a ministerial doctoral fellowship of the French Government. 


\begin{tabular}{|c|c|c|c|}
\hline Parameter & Description & Value (unit) & References \\
\hline$r_{\text {cell }}$ & Cell radius & $7.5 \mu \mathrm{m}$ & \\
\hline$r_{\text {cortex }}$ & Cortex radius & $7.5 \mu m$ & \\
\hline$r_{\text {cytosol }}$ & Cytosol radius & $7.25 \mu \mathrm{m}$ & \\
\hline$r_{\text {lamina }}$ & Lamina radius & $4 \mu m$ & \\
\hline$r_{\text {nucleoplasm }}$ & Nucleoplasm radius & $3.95 \mu \mathrm{m}$ & \\
\hline$t_{\text {cortex }}$ & Cortex thickness & $250 \mathrm{~nm}$ & {$[33]$} \\
\hline$t_{\text {lamina }}$ & Lamina thickness & $50 \mathrm{~nm}$ & {$[48,49]$} \\
\hline$E_{c x, 0}$ & Nominal cortex Young modulus & & {$[33,44]$} \\
\hline$E_{c l, 0}$ & Nominal cytosol Young modulus & & $\begin{array}{l}{\left[\begin{array}{ll}50, & 51,33, \\
44]\end{array}\right.}\end{array}$ \\
\hline$E_{l, 0}$ & Nominal lamina Young modulus & $3000 \mathrm{~Pa}$ & {$[33,44]$} \\
\hline$E_{n p, 0}$ & Nominal nucleoplasm Young modulus & $25 \mathrm{~Pa}$ & $\begin{array}{lll}{[35,} & 36,33, \\
44] & \end{array}$ \\
\hline$E_{c x}$ & Equivalent cortex Young modulus & $9.08 \mathrm{~Pa}$ & \\
\hline$E_{c l}$ & Equivalent cytosol Young modulus & $9.16 \mathrm{~Pa}$ & \\
\hline$E_{l}$ & Equivalent lamina Young modulus & $74.53 \mathrm{~Pa}$ & \\
\hline$E_{n p}$ & Equivalent nucleoplasm Young modu- & $24.38 \mathrm{~Pa}$ & \\
\hline$\nu_{c}$ & Cytoplasm Poisson ratio & 0.3 & {$[33]$} \\
\hline$\nu_{n}$ & Nucleus Poisson ratio & 0.4 & {$[33]$} \\
\hline & Cytosol characteristic time & $1 \mathrm{~s}$ & {$[52,12]$} \\
\hline & Nucleoplasm characteristic time & $2 \mathrm{~s}$ & $\begin{array}{l}{[18,53,36,} \\
12]\end{array}$ \\
\hline & Nucleoplasm plasticity threshold & $4 \mathrm{~Pa}$ & \\
\hline$\rho_{c p}$ & Cytoplasm density & $1000 \mathrm{~kg} / \mathrm{m}^{3}$ & {$[54]$} \\
\hline$\rho_{n}$ & Nucleus density & $1400 \mathrm{~kg} / \mathrm{m}^{3}$ & {$[54]$} \\
\hline$A_{c p, 0}$ & Initial cytoplasm area & $126 \mu m^{2}$ & \\
\hline$A_{c x, 0}$ & Initial cortex area & $11 \mu m^{2}$ & \\
\hline$A_{c l, 0}$ & Initial cytosol area & $115 \mu m^{2}$ & \\
\hline
\end{tabular}




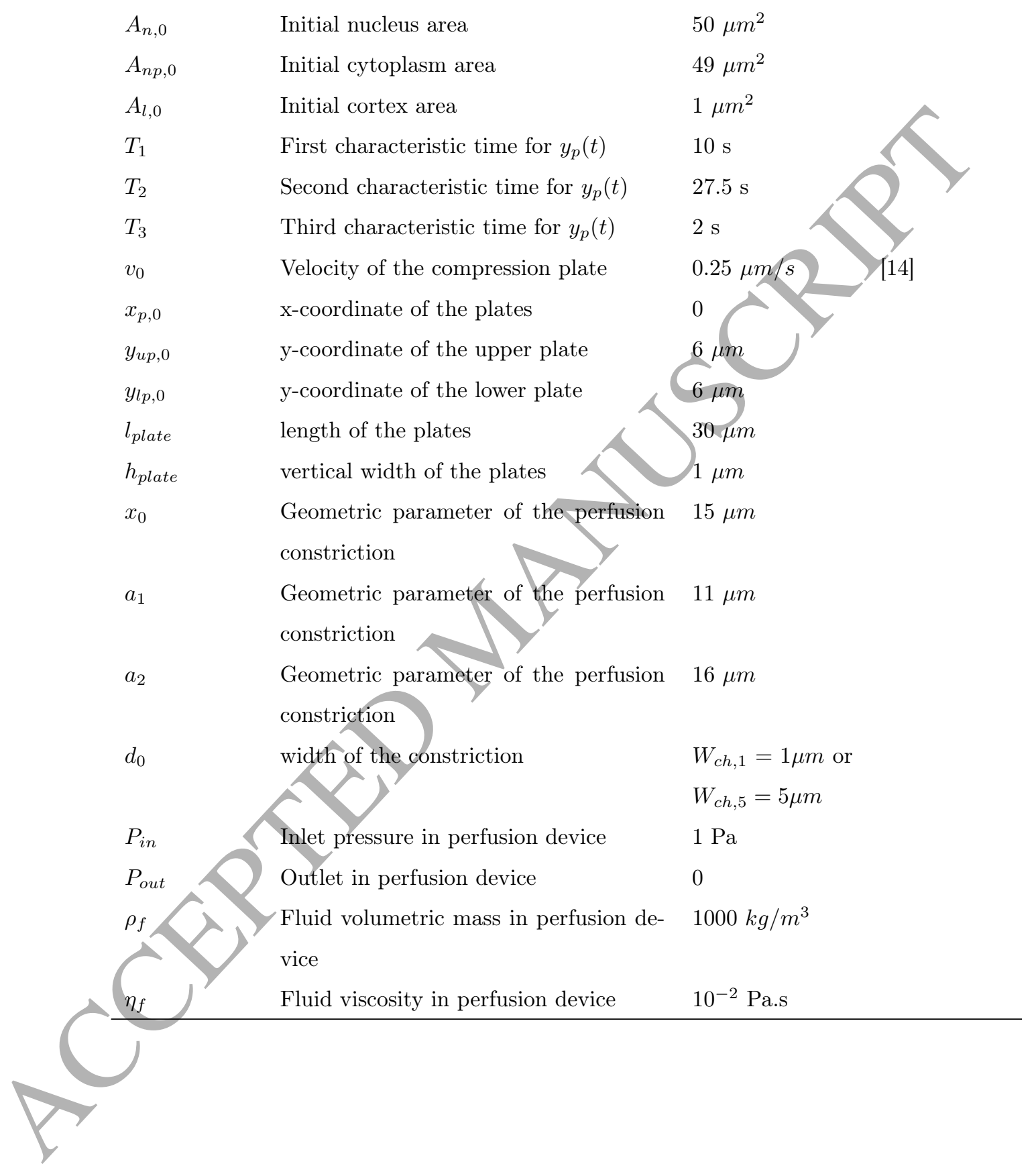




\section{Appendices}

\section{A. Characteristic functions}

Throughout the paper, each cellular component is geometrically defined through a characteristic function $g$, which is a composition of a Heaviside function $h$ and a spatial level set $l$ as follows

$$
g_{i}(\mathbf{x})=h \circ l_{i}(\mathbf{x})= \begin{cases}1 & \text { for } l_{i}(\mathbf{x})>0 \\ 0 & \text { for } l_{i}(\mathbf{x})<0\end{cases}
$$

where $\mathrm{i}=\mathrm{np}, \mathrm{l}, \mathrm{cl}, \mathrm{cx}$. The nucleoplasm and the lamina constitute the nucleus $\left(g_{n}=g_{n p}+g_{l}\right)$, while the cytosol and the cortex constitute the cytoplasm $\left(g_{c}=g_{c l}+g_{c x}\right)$.

B. Nucleus geometry

$$
\begin{aligned}
& g_{l}(\mathbf{x})= \begin{cases}1 & \text { if } r_{\text {nucleoplasm }}^{2}<\left\|\mathbf{x}-\mathbf{c}_{\text {nucleus }}\right\|<r_{\text {lamina }}^{2} \\
0 & \text { otherwise }\end{cases} \\
& g_{n p}(\mathbf{x})= \begin{cases}1 & \text { if }\left\|\mathbf{x}-\mathbf{c}_{\text {nucleus }}\right\|<r_{\text {nucleoplasm }}^{2} \\
0 & \text { otherwise }\end{cases}
\end{aligned}
$$

where $\mathbf{c}_{\text {nucleus }}$ is the position of the center of the nucleus, $r_{\text {nucleoplasm }}$ and $r_{\text {lamina }}$ are the nucleoplasm and lamina radii, respectively and $\mathbf{x}$ is the actual spatial position.

\section{Compression experiment}

The compression plate are defined by the following Heaviside functions

$$
g_{u p}(\mathbf{x}-\mathbf{u})= \begin{cases}1 & \text { if } l_{u p}>0 \\ 0 & \text { otherwise }\end{cases}
$$




$$
g_{l p}(\mathbf{x}-\mathbf{u})= \begin{cases}1 & \text { if } l_{l p}>0 \\ 0 & \text { otherwise }\end{cases}
$$

such that $g_{\text {plate }}=g_{u p}+g_{l p}$, with $l_{u p}$ and $l_{l p}$ two level set functions expressed as

$$
\begin{aligned}
l_{\text {up }} & =-\left(\frac{x-x_{p, 0}}{l_{\text {plate }}}\right)^{8}-\left(\frac{y-y_{\text {up }, 0}-y_{p}(t)}{g_{\text {plate }}}\right)^{4}-1 \\
l_{l p} & =-\left(\frac{x-x_{p, 0}}{l_{\text {plate }}}\right)^{8}-\left(\frac{y+y_{\text {lp }, 0}}{g_{\text {plate }}}\right)^{4}-1
\end{aligned}
$$

where $\left(x_{p, 0}, y_{l p, 0}\right)$ and $\left(x_{p, 0}, y_{u p, 0}\right)$ are coordinates of the two plates, respectively and $y_{p}(t)$ is the vertical displacement applied on $t_{u p}$, the lower plate being fixed, and defined as:

$$
y_{p}(t)= \begin{cases}-v_{0}\left(t-T_{1}\right) & \text { if } T_{1} \leq t \leq T_{1}+T_{2} \\ -v_{0} T_{2} & \text { if } T_{1}+T_{2} \leq t \leq T_{1}+T_{2}+T_{3} \\ -v_{0}\left(-t+T_{1}+2 T_{2}+T_{3}\right) & \text { if } T_{1}+T_{2}+T_{3} \leq t \leq T_{1}+2 T_{2}+T_{3}\end{cases}
$$

with $v_{0}$ the compression velocity and $T_{1}, T_{2}$ and $T_{3}$ the time parameters defining the loading unloading of the plate (see Figure C.14).

\section{Cell geometry}

$$
\begin{gathered}
g_{c x}(\mathbf{x})= \begin{cases}1 & \text { if } r_{\text {cytosol }}^{2}<\left\|\mathbf{x}-\mathbf{c}_{\text {nucleus }}\right\|<r_{\text {cortex }}^{2} \\
0 & \text { otherwise }\end{cases} \\
g_{c l}(\mathbf{x})= \begin{cases}1 & \text { if }\left\|\mathbf{x}-\mathbf{c}_{\text {nucleus }}\right\|<r_{\text {cytosol }}^{2} \\
0 & \text { otherwise }\end{cases}
\end{gathered}
$$

with $r_{\text {cytosol }}^{2}$ and $r_{\text {cortex }}^{2}$ being the cytosol and cortex radii, respectively. 


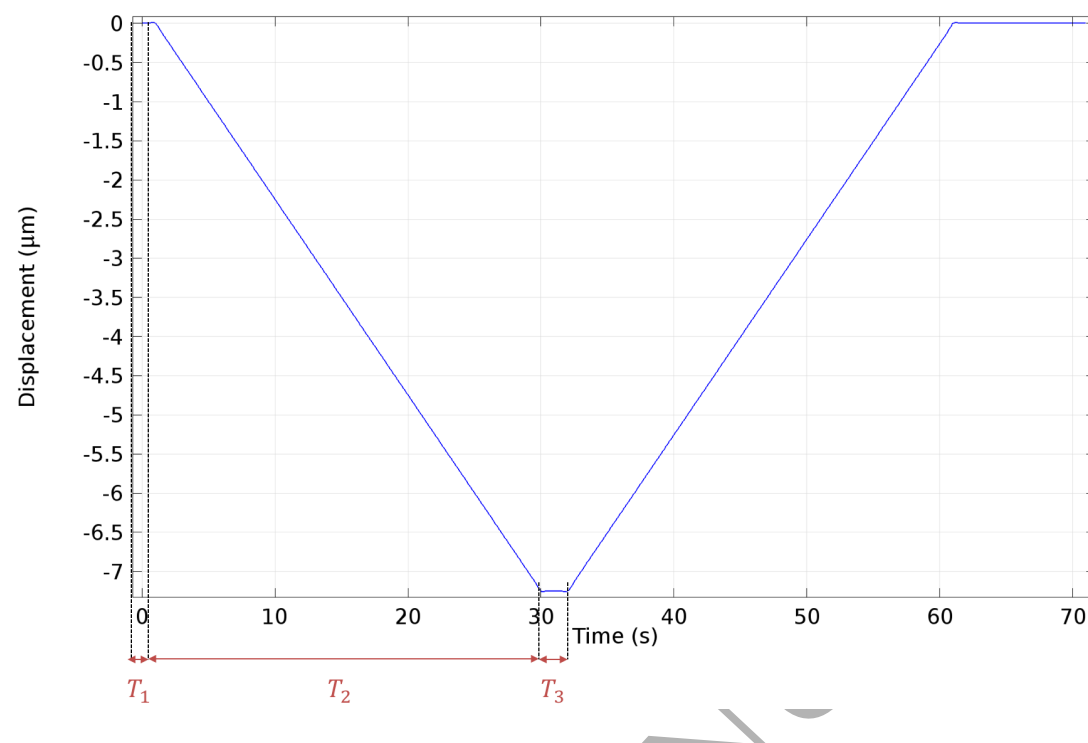

Figure C.14: Motion of the mobile upper plate

\section{E. Perfusion experiment}

E.1. Geometry

$$
\begin{aligned}
& g_{u c}(\mathbf{x}-\mathbf{u})= \begin{cases}1 & \text { if } l_{u c}<0 \\
0 & \text { otherwise }\end{cases} \\
& g_{l c}(\mathbf{x}-\mathbf{u})= \begin{cases}1 & \text { if } l_{l c}<0 \\
0 & \text { otherwise }\end{cases}
\end{aligned}
$$

such the $g_{c h}=g_{u c}+g_{l c}$, where $l_{u c}$ and $l_{l c}$ are two level set functions defined

$$
\begin{aligned}
& l_{u c}=\left(\frac{x-x_{0}}{a_{1}}\right)^{16}+\left(\frac{y-y_{0}}{a_{1}}\right)^{16}+\left(\frac{x-x_{0}-y+y_{0}}{a_{2}}\right)^{16}+\left(\frac{x-x_{0}+y-y_{0}}{a_{2}}\right)^{17} \\
& l_{l c}=\left(\frac{x-x_{0}}{a_{1}}\right)^{16}+\left(\frac{y+y_{0}}{a_{1}}\right)^{16}+\left(\frac{x-x_{0}-y-y_{0}}{a_{2}}\right)^{16}+\left(\frac{x-x_{0}+y+y_{0}}{a_{2}}\right)^{17}
\end{aligned}
$$


where $x_{0}, a_{1}$ and $a_{2}$ are geometric parameters and $y_{0}=a_{1}+d_{0} / 2, d_{0}$ being the width of the constriction.

\section{E.2. Parametric study}

The perfusion model involves 41 parameters (see Table 1), but not all are of the same importance. We can classify them in three categories:

1. The prameters that purely define the system geometry and its dynamics

$$
\begin{aligned}
& \left(r_{\text {cell }}, r_{\text {cortex }}, r_{\text {cytosol }}, r_{\text {lamina }}, r_{\text {nucleoplasm }}, t_{\text {cortex }}, t_{\text {lamina }}, A_{c p, 0}, A_{c l, 0},\right. \\
& A_{c x, 0}, A_{n, 0}, A_{n p, 0}, A_{l, 0} T_{1}, T_{2}, T_{3}, v_{0}, x_{p, 0}, y_{\text {up }, 0}, y_{l p, 0}, l_{\text {plate }}, h_{\text {plate }}, x_{0}, \\
& \left.a_{1}, a_{2}, d_{0}, P_{i n}, P_{\text {out }}, \rho_{f}, \eta_{f}\right)
\end{aligned}
$$

2. The parameters that have been well defined in the literature $\left(\nu_{c}, \nu_{n}, \rho_{c p}\right.$, $\left.\rho_{n}\right)$

3. The parameters that present a high variability $\left(E_{c x, 0}, E_{c l, 0}, E_{l, 0}, E_{n p, 0}\right.$, $\left.\tau_{c l}, \tau_{n p}, s\right)$

We assume that the parameters of the $1^{\text {st }}$ and $2^{\text {nd }}$ categories do not influence the overall results of our work. Among the last category, we chose to focus on those that may play a critical role during the perfusion assay. Considering our interest in the interaction between cytosol and lamina, we chose to perform a parametric study on $E_{c l, 0}, E_{l, 0}$ and $\tau_{c l}$. The plasticity threshold was not included since it was calibrated on the compression test and the value was then kept constant. The sensibility study was conducted on both the wild-type cell model and the lamin-deficient one. For each parameter, 3 simulations were performed using the initial value of the parameter and $\pm 10 \%$. We analyzed the nuclear deviatoric strain $\epsilon_{n}^{D}$ as function of the horizontal displacement of the cell center of inertia $d_{c, x}$ and that said displacement as a function of time in order to assess the influence of cell mechanical parameters on the passage time. 

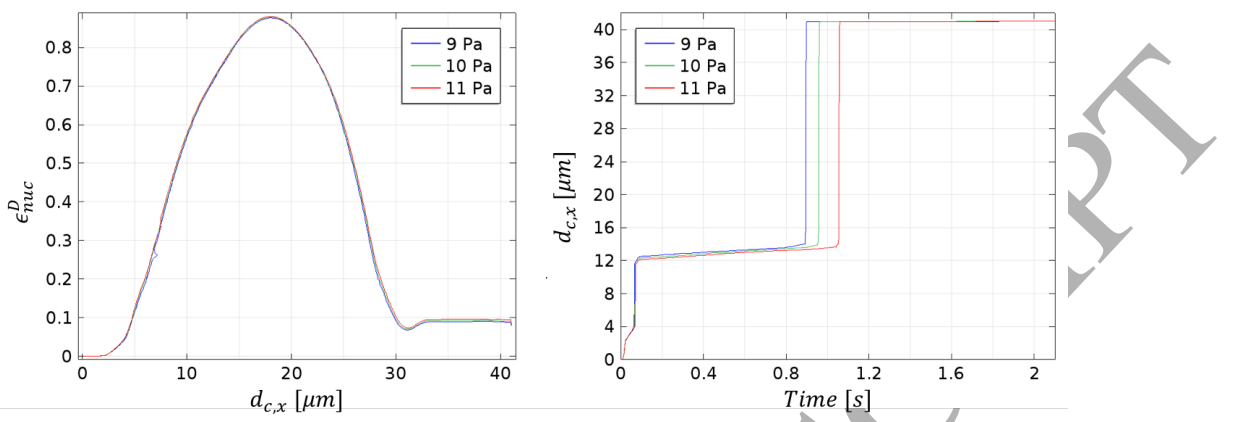

Figure E.15: Parametric study on $E_{c l, 0}$. Left: Evolution of the nuclear deviatoric strain $\boldsymbol{\epsilon}_{n}^{D}$ as function of the horizontal displacement of the cell center of inertia $d_{c, x}$ and Right: Horizontal displacement of the cell center of inertia as a function of time during the $1 \mu \mathrm{m}$-wide micro-channel perfusion simulation for the wild-type model.

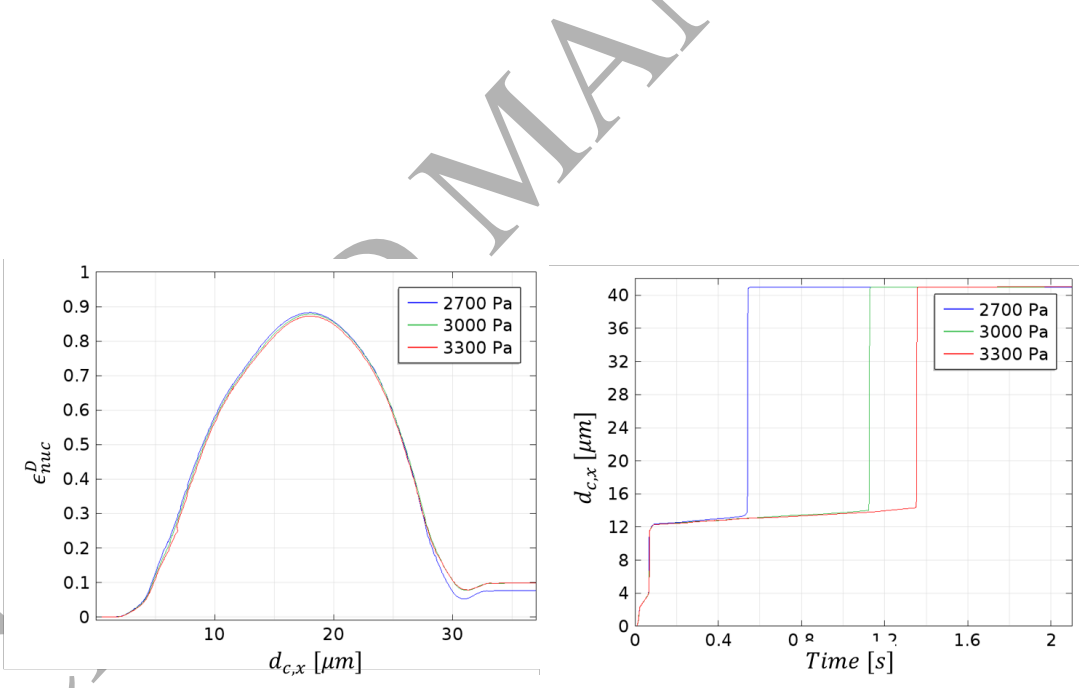

Figure E.16: Parametric study on $E_{l, 0}$. Left: Evolution of the nuclear deviatoric strain $\epsilon_{n}^{D}$ as function of the horizontal displacement of the cell center of inertia $d_{c, x}$ and Right: Horizontal displacement of the cell center of inertia as a function of time during the $1 \mu \mathrm{m}$-wide micro-channel perfusion simulation for the wild-type model. 

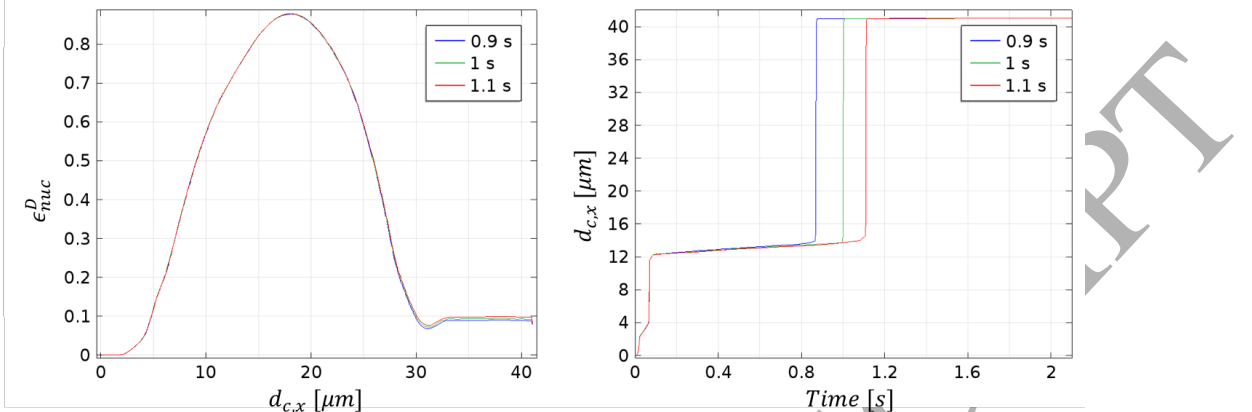

Figure E.17: Parametric study on $\tau_{c l}$. Left: Evolution of the nuclear deviatoric strain $\boldsymbol{\epsilon}_{n}^{D}$ as function of the horizontal displacement of the cell center of inertia $d_{c, x}$ and Right: Horizontal displacement of the cell center of inertia as a function of time during the $1 \mu m$-wide micro-channel perfusion simulation for the wild-type model.
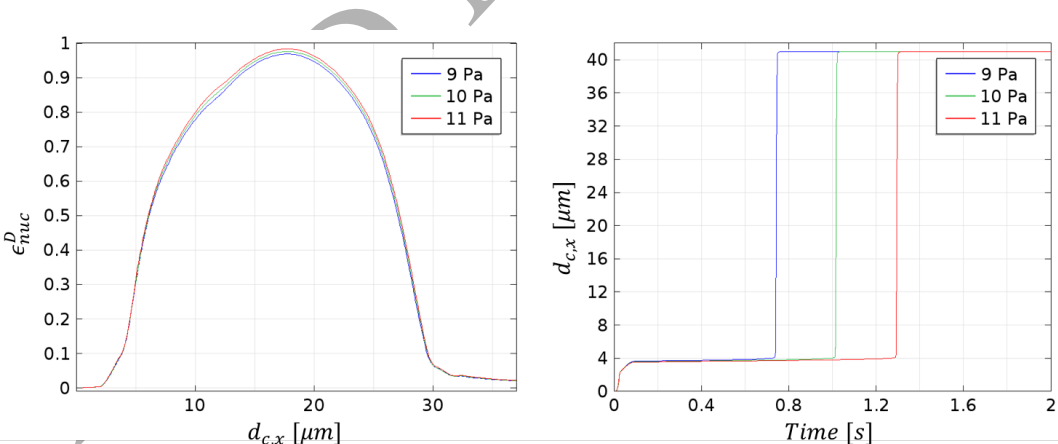

Figure E.18: Parametric study on $E_{c l, 0}$. Left: Evolution of the nuclear deviatoric strain $\epsilon_{n}^{D}$ as function of the horizontal displacement of the cell center of inertia $d_{c, x}$ and Right: Horizontal displacement of the cell center of inertia as a function of time during the $1 \mu \mathrm{m}$-wide micro-channel perfusion simulation for the lamin-deficient model. 

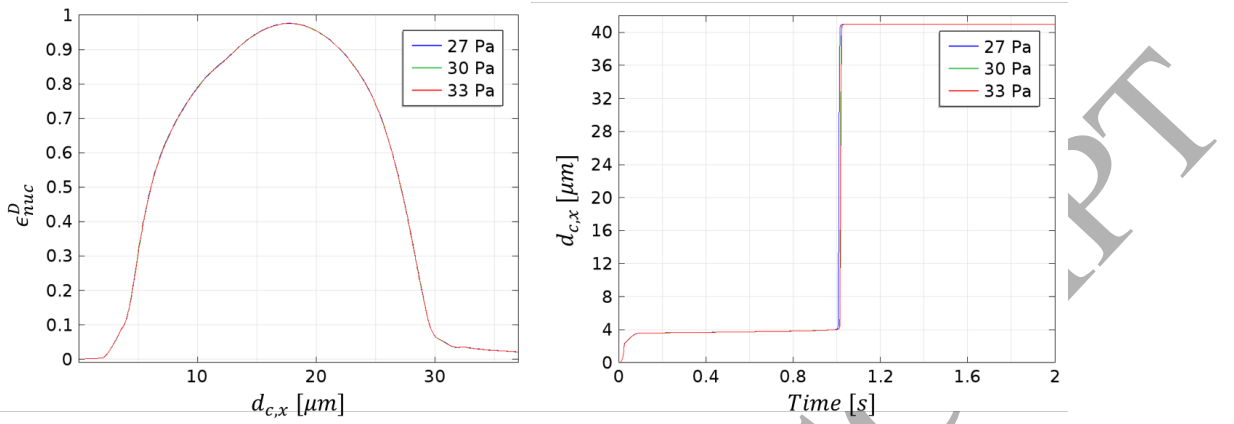

Figure E.19: Parametric study on $E_{l, 0}$. Left: Evolution of the nuclear deviatoric strain $\boldsymbol{\epsilon}_{n}^{D}$ as function of the horizontal displacement of the cell center of inertia $d_{c, x}$ and Right: Horizontal displacement of the cell center of inertia as a function of time during the $1 \mu \mathrm{m}$-wide micro-channel perfusion simulation for the lamin-deficient model.

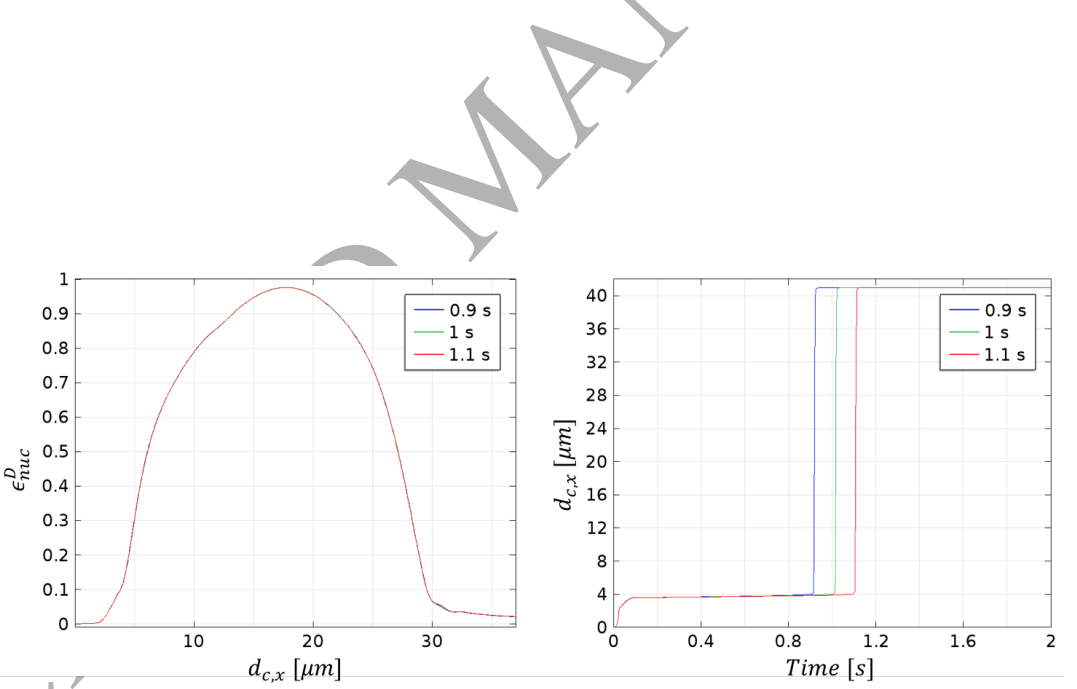

Figure E.20: Parametric study on $\tau_{c l}$. Left: Evolution of the nuclear deviatoric strain $\boldsymbol{\epsilon}_{n}^{D}$ as function of the horizontal displacement of the cell center of inertia $d_{c, x}$ and Right: Horizontal displacement of the cell center of inertia as a function of time during the $1 \mu \mathrm{m}$-wide micro-channel perfusion simulation for the lamin-deficient model. 


\section{References}

[1] C. L. Chaffer, R. A. Weinberg, A Perspective on Cancer Cell Metastasis, Science 331 (2011) 1559-1564.

[2] M. Zwerger, C. Y. Ho, J. Lammerding, Nuclear Mechanics in Disease, Annual Review of Biomedical Engineering 13 (2011) 397-428.

[3] K. Wolf, P. Friedl, Molecular mechanisms of cancer cell invasion and plasticity, The British Journal of Dermatology 154 Suppl 1 (2006) 11-15.

[4] P. Friedl, K. Wolf, J. Lammerding, Nuclear mechanics during cell migration, Current Opinion in Cell Biology 23 (2011) $55-64$.

[5] C. Schwartz, M. Fischer, K. Mamchaoui, A. Bigot, T. Lok, C. Verdier, A. Duperray, R. Michel, I. Holt, T. Voit, S. Quijano-Roy, G. Bonne, C. Coirault, Lamins and nesprin-1 mediate inside-out mechanical coupling in muscle cell precursors through FHOD1, Scientific Reports 7 (2017) 1253.

[6] C. T. Skau, R. S. Fischer, P. Gurel, H. R. Thiam, A. Tubbs, M. A. Baird, M. W. Davidson, M. Piel, G. M. Alushin, A. Nussenzweig, P. S. Steeg, C. M. Waterman, FMN2 Makes Perinuclear Actin to Protect Nuclei during Confined Migration and Promote Metastasis, Cell 167 (2016) 1571-1585.e18.

[7] K. Wolf, M. t. Lindert, M. Krause, S. Alexander, J. t. Riet, A. L. Willis, R. M. Hoffman, C. G. Figdor, S. J. Weiss, P. Friedl, Physical limits of cell migration: Control by ECM space and nuclear deformation and tuning by proteolysis and traction force, The Journal of Cell Biology 201 (2013) 1069-1084.

[8] C. Y. Ho, J. Lammerding, Lamins at a glance, J Cell Sci 125 (2012) 2087-2093.

[9] A. C. Rowat, J. Lammerding, J. H. Ipsen, Mechanical Properties of the Cell Nucleus and the Effect of Emerin Deficiency, Biophysical Journal 91 (2006) 4649-4664. 
[10] C. T. Lim, E. H. Zhou, S. T. Quek, Mechanical models for living cells-a review, Journal of Biomechanics 39 (2006) 195-216.

[11] P. Isermann, P. M. Davidson, J. D. Sliz, J. Lammerding, Assays to measure nuclear mechanics in interphase cells, Current protocols in cell biology editorial board, Juan S. Bonifacino ... [et al.] CHAPTER (2012) Unit22.16.

[12] F. Guilak, J. R. Tedrow, R. Burgkart, Viscoelastic properties of the cell nucleus, Biochemical and Biophysical Research Communications 269 (2000) $781-786$.

[13] M. Monticelli, D. V. Conca, E. Albisetti, A. Torti, P. P. Sharma, G. Kidiyoor, S. Barozzi, D. Parazzoli, P. Ciarletta, M. Lupi, D. Petti, R. Bertacco, Magnetic domain wall tweezers: a new tool for mechanobiology studies on individual target cells, Lab Chip 16 (2016) 2882-2890.

[14] N. Caille, O. Thoumine, Y. Tardy, J.-J. Meister, Contribution of the nucleus to the mechanical properties of endothelial cells, Journal of Biomechanics 35 (2002) 177-187.

[15] M. L. Lombardi, M. Zwerger, J. Lammerding, Biophysical Assays to Probe the Mechanical Properties of the Interphase Cell Nucleus: Substrate Strain Application and Microneedle Manipulation, Journal of Visualized Experiments : JoVE (2011).

[16] M. M. Nava, M. T. Raimondi, R. Pietrabissa, Bio-chemo-mechanical models for nuclear deformation in adherent eukaryotic cells, Biomechanics and Modeling in Mechanobiology 13 (2014) 929-943.

[17] F. Erdel, M. Baum, K. Rippe, The viscoelastic properties of chromatin and the nucleoplasm revealed by scale-dependent protein mobility, Journal of Physics: Condensed Matter 27 (2015) 064115.

[18] A. H. B. de Vries, B. E. Krenn, R. van Driel, V. Subramaniam, J. S. Kanger, Direct Observation of Nanomechanical Properties of Chromatin in Living Cells, Nano Letters 7 (2007) 1424-1427. 
[19] H. W. Hou, Q. S. Li, G. Y. H. Lee, A. P. Kumar, C. N. Ong, C. T. Lim, Deformability study of breast cancer cells using microfluidics, Biomedical Microdevices 11 (2009) 557-564.

[20] Y. Luo, D. Chen, Y. Zhao, C. Wei, X. Zhao, W. Yue, R. Long, J. Wang, J. Chen, A constriction channel based microfluidic system enabling continuous characterization of cellular instantaneous Young's modulus, Sensors and Actuators B: Chemical 202 (2014) 1183-1189.

[21] K. N. Dahl, A. J. Engler, J. D. Pajerowski, D. E. Discher, Power-law rheology of isolated nuclei with deformation mapping of nuclear substructures, Biophysical Journal 89 (2005) 2855-2864.

[22] H. Liu, J. Wen, Y. Xiao, J. Liu, S. Hopyan, M. Radisic, C. A. Simmons, Y. Sun, In Situ Mechanical Characterization of the Cell Nucleus by Atomic Force Microscopy, ACS Nano 8 (2014) 3821-3828.

[23] K. Tomankova, P. Kolar, J. Malohlava, H. Kolarova, Mechanical characterisation of HeLa cells using atomic force microscopy, Current Microscopy Contributions to Advances in Science and Technology. Badajoz: Formatex Research Center (2012) 549-554.

[24] A. Rowat, L. Foster, M. Nielsen, M. Weiss, J. Ipsen, Characterization of the elastic properties of the nuclear envelope, Journal of the Royal Society Interface 2 (2005) 63-69.

[25] J. Swift, 1. L. Ivanovska, A. Buxboim, T. Harada, P. C. D. P. Dingal, J. Pinter, J. D. Pajerowski, K. R. Spinler, J.-W. Shin, M. Tewari, F. Rehfeldt, D. W. Speicher, D. E. Discher, Nuclear Lamin-A Scales with Tissue Stiffness and Enhances Matrix-Directed Differentiation, Science 341 (2013) 1240104 .

[26] J. Swift, D. E. Discher, The nuclear lamina is mechano-responsive to ECM elasticity in mature tissue, Journal of Cell Science (2014) jcs.149203. 
[27] Y. Gruenbaum, R. D. Goldman, R. Meyuhas, E. Mills, A. Margalit, A. Fridkin, Y. Dayani, M. Prokocimer, A. Enosh, The Nuclear Lamina and Its Functions in the Nucleus, International Review of Cytology 226 (2003) $1-62$.

[28] J. D. Pajerowski, K. N. Dahl, F. L. Zhong, P. J. Sammak, D. E. Discher, Physical plasticity of the nucleus in stem cell differentiation, Proceedings of the National Academy of Sciences of the United States of America 104 (2007) 15619-15624.

[29] S. Deguchi, K. Maeda, T. Ohashi, M. Sato, Flow-induced hardening of endothelial nucleus as an intracellular stress-bearing organelle, Journal of Biomechanics 38 (2005) 1751-1759.

[30] A. D. Stephens, E. J. Banigan, S. A. Adam, R. D. Goldman, J. F. Marko, Chromatin and lamin A determine two different mechanical response regimes of the cell nucleus, Molecular Biology of the Cell (2017) mbc.E16-09-0653.

[31] Y. Ujihara, M. Nakamura, S. Wada, A Mechanical Cell Model and Its Application to Cellular Biomechanics, INTECH Open Access Publisher, 2011.

[32] C. Giverso, A. Grillo, L. Preziosi, Influence of nucleus deformability on cell entry into cylindrical structures, Biomechanics and Modeling in Mechanobiology 13 (2014) 481-502.

[33] D. Aubry, H. Thiam, M. Piel, R. Allena, A computational mechanics approach to assess the link between cell morphology and forces during confined migration, Biomechanics and Modeling in Mechanobiology 14 (2014) 143-157.

[34] A. Vaziri, A. Gopinath, V. S. Deshpande, Continuum-based computational models for cell and nuclear mechanics, Journal of the Mechanics of Materials and Structures 2 (2007) 1169-1192. 
[35] A. Vaziri, H. Lee, M. K. Mofrad, Deformation of the cell nucleus under indentation: Mechanics and mechanisms, Journal of Materials Research 21 (2006) 2126-2135.

[36] A. Vaziri, M. R. K. Mofrad, Mechanics and deformation of the nucleus in micropipette aspiration experiment, Journal of Biomechanics 40 (2007) 2053-2062.

[37] X. Cao, E. Moeendarbary, P. Isermann, P. M. Davidson, X. Wang, M. B. Chen, A. K. Burkart, J. Lammerding, R. D. Kamm, V. B. Shenoy, A Chemomechanical Model for Nuclear Morphology and Stresses during Cell Transendothelial Migration, Biophysical Journal 111 (2016) 1541-1552.

[38] F. Y. Leong, Q. Li, C. T. Lim, K.-H. Chiam, Modeling cell entry into a micro-channel, Biomechanics and Modeling in Mechanobiology 10 (2011) $755-766$.

[39] R. M. Christensen, Mechanics of compósite materials, Wiley, New York, 1979.

[40] J. Donea, A. Huerta, J.-P. Ponthot, A. Rodriguez-Ferran, Arbitrary Lagrangian-Eulerian Methods, in: Encyclopedia of Computational Mechanics, John Wiley \& Sons, Ltd, 2004.

[41] T. Belytschko, W. K. Liu, B. Moran, Nonlinear finite elements for continua and structures, Wiley, 2000.

[42] T. Harada, J. Swift, J. Irianto, J.-W. Shin, K. R. Spinler, A. Athirasala, R. Diegmiller, P. D. P. Dingal, I. L. Ivanovska, D. E. Discher, Nuclear lamin stiffness is a barrier to $3 \mathrm{~d}$ migration, but softness can limit survival, The Journal of Cell Biology 204 (2014) 669-682.

[43] A. L. McGregor, C.-R. Hsia, J. Lammerding, Squish and squeeze the nucleus as a physical barrier during migration in confined environments, Current Opinion in Cell Biology 40 (2016) 32-40. 
[44] R. Allena, Mechanical modelling of confined cell migration across constricted-curved micro-channels, Molecular \& cellular biomechanics: MCB 11 (2014) 185-208.

[45] J. L. Philipp Isermann, Nuclear mechanics and mechanotransduction in health and disease., Current biology : CB 23 (2013) R1113-21.

[46] R. Allena, D. Aubry, 'Run-and-tumble' or 'look-and-run'? A mechanical' model to explore the behavior of a migrating amoeboid cell, Journal of Theoretical Biology 306 (2012) 15-31.

[47] D. Aubry, S. Deveraux, R. Allena, Cell nucleus membrane modeling through shell tangential differential calculus, in: Oral presentation at the 7th European Congress on Computational Methods in Applied Sciences and Engineering, 2016.

[48] J. M. Bridger, N. Foeger, I. R. Kill, H. Herrmann, The nuclear lamina, FEBS Journal 274 (2007) 1354-1361.

[49] A. Kaminski, G. R. Fedorchak, J. Lammerding, The cellular mastermind mechanotransduction and the nucleus, Progress in molecular biology and translational science 126 (2014) 157-203.

[50] A. Kamgoue, J. Ohayon, P. Tracqui, Estimation of Cell Young's Modulus of Adherent Cells Probed by Optical and Magnetic Tweezers: Influence of Cell Thickness and Bead Immersion, Journal of Biomechanical Engineering 129 (2007) 523 .

[51] Y. A. Ayala, B. Pontes, D. S. Ether, L. B. Pires, G. R. Araujo, S. Frases, L. F. Romao, M. Farina, V. Moura-Neto, N. B. Viana, H. M. Nussenzveig, Rheological properties of cells measured by optical tweezers, BMC Biophysics 9 (2016) 5 .

[52] J. S. Milner, M. W. Grol, K. L. Beaucage, S. J. Dixon, D. W. Holdsworth, Finite-Element Modeling of Viscoelastic Cells During High-Frequency Cyclic Strain, Journal of Functional Biomaterials 3 (2012) 209-224. 
[53] A. Celedon, C. M. Hale, D. Wirtz, Magnetic Manipulation of Nanorods in the Nucleus of Living Cells, Biophysical Journal 101 (2011) 1880-1886.

[54] R. Milo, R. Phillips, Cell Biology by the Numbers, Garland Science, 2015.

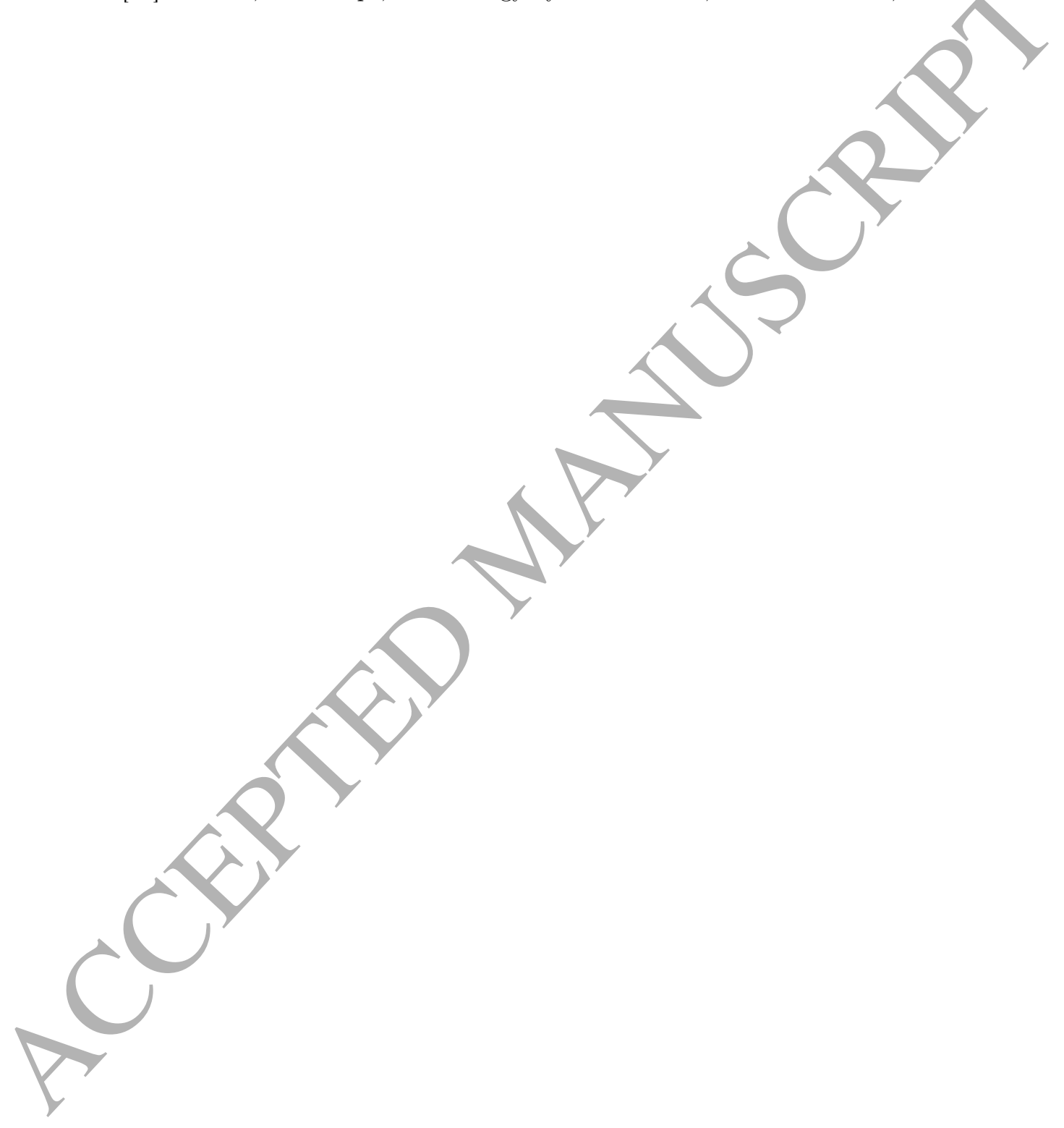

